\title{
Factors Determining City Brand Equity-A Systematic Literature Review
}

\author{
Hanna Górska-Warsewicz \\ Department of Food Market and Consumer Research, Institute of Human Nutrition Sciences, \\ Warsaw University of Life Sciences (SGGW-WULS), 159C Nowoursynowska Street, 02-787 Warsaw, Poland; \\ hanna_gorska_warsewicz@sggw.pl; Tel.: +48-22-59-37-144
}

Received: 30 August 2020; Accepted: 18 September 2020; Published: 23 September 2020

check for updates

\begin{abstract}
The aim of this study is to analyze factors determining city brand equity (CBE) on the bases of a systematic literature review (SLR) according to a procedure developed by Tranfield. Five databases were searched (Scopus, Web of Sciences, Google Scholar, EBSCO, and Elsevier) for studies containing the term 'city brand equity'. In addition, databases were searched for 'destination brand equity' and 'place brand equity' statements focusing on city brands. This SLR contains 36 empirical studies and does not include conference materials, and books. The analysis was based on three issues: general information (author/authors, year of publication, research tools, sample size, city and country as well as sample population), research specification (variables, hypotheses or research questions, statistical methods and research tools), and findings related to CBE and practical implications for destination marketing organizations, and destination or city managers. In summary, there are many factors determining CBE, including brand image, brand quality and brand awareness. These factors are related to cultural, environmental and ecological elements, as well as infrastructure and services offered to tourists. Brand assets related to the perception of the city brands in various aspects also proved to be important.
\end{abstract}

Keywords: city brand equity; destination brand equity; place brand equity; city branding

\section{Introduction}

City brand equity (CBE) (CBE—city brand equity, BE—brand equity, CBBE—consumer-based brand equity, SLR-systematic literature review) is a new concept resulting from brand equity [1-5] and city branding [6-15]. It reflects the growing role of the brand in the promotion of tourist destinations [16-18], including cities, and the application of methods and tools used so far by corporate, product and service brands [19-22]. At the same time, city branding is becoming a strategic asset in communicating the city value and achieving a competitive position [12]. An important aspect is also the issue of consumer choice, understood as the destination brand choice model. In this case the city is one of the existing destinations [23].

The concept of BE has been described in the literature as a marketing tool since the 1990s. One of the first definitions proposed by Farquhar [24] points to BE as an added value to the product through the brand image perceived by customers. The frequently quoted definition by Aaker [25] refers to a set of brand assets and liabilities associated with the name and symbol of an organization that add or subtract from the value provided by a product or service. BE is also analyzed from the perspective of the consumer as consumed-based brand equity (CBBE) and defined by Keller [26] in the context of a differential effect that brand knowledge has on consumer response to the marketing of that brand. In this regard, BE means value for customers considered in terms of satisfaction, and trust, as well as value for the company considered in terms of marketing programs, and competitive advantages [25]. Subsequent definitions analyzed different aspects of $\mathrm{BE}$, such as the selection between a product with 
or without a brand, assuming the same level of features [27], the benefits obtained by the consumers from the product and services with a positive impact on the financial results of the company [28], and, in the financial approach, additional cash flow generated by branded products compared to non-branded ones [29].

BE is not a simple construction. Aaker listed five components of BE, i.e., brand loyalty, brand awareness, perceived quality, brand associations, and other proprietary brand assets [25]. Keller, on the other hand, highlighted brand knowledge including brand awareness and brand associations. He proposed four complex elements: brand identity, including brand salience and brand awareness; brand meaning, with brand performance and brand imagery, referring to brand associations; brand responses including consumer feeling and judgments; as well as relationships with brand related to brand loyalty [26]. These two concepts of $\mathrm{BE}$ became the basis for further consideration and practical analysis concerning selected categories of products $[5,30,31]$, and services [32,33]. For example, brand loyalty, perceived quality, brand awareness, brand associations were identified in the study of 12 brands in three product categories (athletic shoes, film for cameras, and color television sets) [34]; six brands in two product categories (cars and televisions) [35]; and brands available in the Chinese sportswear category [5]. BE was also analyzed in relation to the hospitality industry as hotel BE [36-44], the catering industry as restaurant $\mathrm{BE}[43,45-47]$, and the airline industry as airline BE [48-50]. The following elements were identified in the hotel BE research: brand loyalty [38,40,43,51-53], brand awareness [38,40,42,52,54], brand associations [54], perceived quality $[38,40,43,51-53]$, brand image [38,40,42,43,51-53]. Specific elements of the BE were also pointed out, i.e., corporate social responsibility [55], brand satisfaction [56], physical quality [42], staff behavior [42], as well as information and communication technology [57]. Research on restaurant services identified the following elements of restaurant BE: brand awareness [45-47,58], brand image [46,58], brand loyalty [47,58], brand association [45,47], quality expressed as brand quality [46], perceived quality [47,58], as well as food and service quality [45]. Brand trust, brand reputation [45], and green activities were also pointed as elements of restaurant BE [59].

Based on the above arguments, the aim of this study is to analyze the CBE based on a systematic literature review (SLR) according to a three-stage process developed by Tranfield et al.: planning, execution and reporting, and dissemination [60]. This CBE-related research fills a gap in city branding scientific literature, combining issues of $\mathrm{BE}$ and city marketing/city branding. It is also a contribution to the literature due to the lack of SLR studies related to CBE. Earlier SLRs refer to city branding. For example, one study on a systematic literature review from 1988-2014 analyzed four themes, including city branding concept, processes and measurement, branding strategies, social urbanism, and branding culture and tourism [61]. Another study focused on publications from 2005-2016 and analyzed brand dimensions/elements such as negative and positive assets, tangible and intangible elements, physical and environment, culture, activities and facilities as well as psychological elements [62]. The next study concerns publications from 1988-2010 and presents a matrix of two dimensions. The first is the perspective of the place (destination, territory, location and area), city (municipality and town), and city regions (metropolitan, cosmopolitan and urban). The second dimension refers to the marketing approach in terms of promotion, selling, communication, etc., and the brand focused on visual identity, image branding, profile, etc. [63].

The structure of this study is as follows: the first section is an introduction to the analyzed issue. The second one discusses the methodology with the specification of study design, planning the SLR, executing the SLR, and presenting the results. In the third section, the results are presented in three parts: general information, research specifications, general findings, and practical implications. The next section presents a discussion of the results concerning the CBE issue and the main factors determining the equity of the city brands. The last section presents the conclusions, limitations, and proposal of future research. 


\section{Methodology}

\subsection{Study Design}

An SLR was applied as a research method, known and widely used in various scientific areas [64-69]. This study uses a method developed by Tranfield [60], applied in scientific research regarding, among others, tourism research on sustainability [64], bio, green and circular economy trends [70], process mining techniques [71], lean supply chain strategy [72], and sustainability-oriented innovation [73]. The SLR proposed by Tranfield consists of three stages: planning, executing, and reporting/disseminating. Below is a description of their application for the purpose of the literature review on CBE.

\subsection{Planning the $S L R$}

Based on the aim of this study relating to the CBE analysis, the following research questions were formulated:

- What factors influence CBE?

- Is sustainability analyzed as an element of CBE?

- What is the relationship between CBE and sustainability?

In order to answer the research questions, the databases were searched using the term 'city brand equity' in the title, abstract, and as keywords. The 'place brand equity' and 'destination brand equity' searches were also used to check whether the place or destination referred to the city.

The search was conducted on the following databases: Scopus, Web of Sciences, Google Scholar, EBSCO, and Elsevier. The snowball method was also used in two variants: as a backward snowball method consisting of checking reference lists in published articles and a forward snowball method consisting of identifying new tests quoting articles in SLR [74].

\subsection{Executing the SLR}

The databases were searched between 20th and 30th June 2020. The following inclusion criteria were applied: studies based on empirical research, studies describing CBE, studies describing destination or place $B E$ in relation to cities, peer-reviewed papers. No time limits were applied, but the search was limited to publications in English.

Exclusion criteria were applied, including studies describing BE related to countries, tourist areas, not related to cities, studies with theoretical models, practical studies of tourist organizations, a Ph.D. thesis, short reports, rankings of cities, workshop papers, work-in-progress papers, editorials, practice guidelines, book chapters, book reviews, and conference publications: proceedings, posters, abstracts, and others.

The following search strategies were applied:

- Scopus: TITLE-ABS - KEY (city AND brand AND equity), and (TITLE-ABS—KEY (city AND (brand AND equity)); TITLE-ABS—KEY (destination AND brand AND equity), and (TITLE-ABS -KEY (destination AND (brand AND equity)); TITLE-ABS—KEY (place AND brand AND equity), and (TITLE-ABS - KEY (place AND (brand AND equity)); TITLE-ABS-KEY (city AND brand AND equity) AND (LIMIT-TO (DOCTYPE, “ar"));

- Web of Sciences: ALL FIELDS: (city) AND ALL FIELDS: (brand) AND ALL FIELDS: (equity); ALL FIELDS: (place) AND ALL FIELDS: (brand) AND ALL FIELDS: (equity); ALL FIELDS: (destination) AND ALL FIELDS: (brand) AND ALL FIELDS: (equity);

- Google Scholar: allintitle: city brand equity; with the statement: "city brand equity"; allintitle: destination brand equity; with the statement: "destination brand equity"; allintitle: place brand equity; with the statement: "place brand equity"

- EBSCO: city AND brand AND equity; destination AND brand AND equity; place AND brand AND equity 
- Elsevier: Title, abstract, keywords: "city brand equity"; Title, abstract, keywords: "destination brand equity"; Title, abstract, keywords: "place brand equity"

In each case, a three-stage search area was used, first CBE and then the destination, and place BE, including checking if destination or place includes a city. As a result of the applied search strategy, 708 studies were obtained in the 1st stage, 2183 in the 2nd stage, and 938 studies in the 3rd stage. Titles and abstracts were initially analyzed for relevance, appropriateness, and quality of studies. Attention was paid to whether the studies refer to the city with the destination or place search criteria used. All other exclusion criteria were then applied. The backward and forward snowball method was also used. As a result of the strategy described above, 36 studies were obtained.

\subsection{Presenting the $S L R$}

The list of all 36 studies included in the SLR is presented in the (Table S1). The analysis of the studies is presented in 3 parts: general information (author/authors, year of publication, research tools, sample size, city and country as well as sample population) (Table 1), research specification (variables, hypotheses or research questions, statistical methods and research tools) (Table 2), and findings and practical implications (findings and conclusions related to CBE as well as practical implications for destination marketing organizations, destination or city managers and others) (Table 3). 
Table 1. General information related to studies included in the systematic literature review (SLR).

\begin{tabular}{|c|c|c|c|c|c|}
\hline Article & Author, Year & Type of Study & Research Tools/Size of Sample & City/Country & Sample Population \\
\hline [75] & $\begin{array}{l}\text { Huerta-Alvarez, } \\
\text { Cambra-Fierro, } \\
\text { Fuentes-Blasco, } 2020\end{array}$ & Survey & $\begin{array}{l}300 \text { self-administered questionnaires } \\
\text { distributed among guests staying in 3-, } \\
4 \text { - and 5-star hotels }\end{array}$ & Lima, Peru & $\begin{array}{c}\text { Nationality: } \\
\text { Gender: female }(47.7 \%), \text { male }(52.3 \%) \\
\text { Age: under } 35 \text { years of age }(47 \%) \text {, over } 35(53 \%)\end{array}$ \\
\hline [76] & $\begin{array}{c}\text { Dedeoglu, Niekerk, } \\
\text { Weinland, Celuch, } 2019\end{array}$ & Survey & $\begin{array}{l}450 \text { questionnaires obtained by personal } \\
\text { interviews and } 200 \text { questionnaires } \\
\text { obtained by drop-and-collect method; } \\
478 \text { appropriate for data analysis }\end{array}$ & Alanya, Turkey & $\begin{array}{l}\text { Nationality: German }(31.6 \%) \text {, Russian }(21.1 \%) \\
\text { Gender: female }(51.4 \%) \text {, male }(48.6 \%) \\
\text { Age: under } 58(48.1 \%), 58 \text { years of age or older }(51.9 \%)\end{array}$ \\
\hline [77] & $\begin{array}{l}\text { Tran, Nguyen, Tran, Tran, } \\
\text { Huynh, } 2019\end{array}$ & Survey & 319 questionnaires analyzed & Hoi An, Vietnam & $\begin{array}{l}\text { Gender: male }(41.1 \%) \text {, female }(58.9 \%) \\
\text { Age: under } 40(71.1 \%) \text {, over } 40(28.9 \%)\end{array}$ \\
\hline [78] & $\begin{array}{l}\text { Tarman, Soleh, Ari, } \\
\text { Rahmat, } 2019\end{array}$ & Survey & 265 respondents & $\begin{array}{l}\text { Tasikmalaya, Indonesia } \\
\text { (known as Crafts City) }\end{array}$ & $\begin{array}{c}\text { Nationality: native of the city }(55.8 \%) \\
\text { Gender: female }(58.1 \%), \text { male }(41.9 \%) \\
\text { Age: below } 25(40.0 \%), 25-34(18.3 \%), 35-44(35.3 \%), \\
45-54(30.0 \%)\end{array}$ \\
\hline [79] & Rani, 2019 & Survey & 150 questionnaires & Kurukshetra, India & $\begin{array}{c}\text { Gender: female }(12.7 \%) \text {, male }(87.3 \%) \\
\text { Age: } 18-23(78.1 \%)\end{array}$ \\
\hline [80] & $\begin{array}{l}\text { Subaqyo, Ujianto, } \\
\text { Susanti, } 2019\end{array}$ & Survey & 250 questionnaires & Banyuwangi, Indonesia & not specified \\
\hline [81] & $\begin{array}{l}\text { Gomez, Fernandez, Molina, } \\
\text { Aranda, } 2018\end{array}$ & Online survey & $\begin{array}{l}225 \text { respondents randomly assigned; } \\
\text { restrictions: tourists visited } 2 \text { or more } \\
\text { European capitals in the past } 5 \text { years; one } \\
\text { journeys in the previous year; minimum } \\
\text { of } 2 \text { nights at each destination; leisure } \\
\text { travels; travelling experience: more than } \\
\qquad 2 \text { trips per year }\end{array}$ & $\begin{array}{l}\text { London, UK } \\
\text { Paris, France } \\
\text { Berlin, Germany } \\
\text { Rome, Italy } \\
\text { Madrid, Spain }\end{array}$ & $\begin{array}{c}\text { number of responses per city was distributed as } \\
\text { follows: London: 55; Paris: } 41 \text {; Berlin: 30; Rome: 33; } \\
\text { and Madrid: } 60 \\
\text { male }(47.3 \%), \text { female }(52.7 \%) \\
\text { age: } 15-24(18.2 \%), 25-34(36.4 \%), 35-44(21.8 \%) \\
45-54(10.9 \%) \text {, over } 55(12.7 \%)\end{array}$ \\
\hline [82] & Kotsi, Pike, Gottlieb, 2018 & Survey & $\begin{array}{l}1200 \text { questionnaires distributed in France } \\
\text { and Australia; } 591 \text { completions were } \\
\text { achieved in Australia, along with } 600 \text { in } \\
\text { France, including } 403 \text { useable responses } \\
\text { from Australia and } 365 \text { from France }\end{array}$ & Dubai, United Arab Emirates & $\begin{array}{c}\text { Nationality: French (365) and Australian (403) } \\
\text { France: male (53.7\%), female, 46.3\%; age: } 18-25 \\
(13.7 \%), 26-34(15.9 \%), 35-49(22.7 \%), 50-64(27.1 \%), \\
65+(20.5 \%) \\
\text { Australia male }(48.4 \%) \text {, female }(51.6 \%) \text {; age: } 18-25 \\
(6.9 \%), 26-34(10.9 \%), 35-49(16.9 \%), 50-64(33.0 \%), \\
65+(32.3 \%)\end{array}$ \\
\hline [83] & $\begin{array}{l}\text { Duman, Ozbal, } \\
\text { Duerod, } 2018\end{array}$ & Survey & $\begin{array}{l}\text { 3,000 online questionnaires and } 400 \\
\text { paper copies distributed, } 479 \text { accepted } \\
\text { for analysis }\end{array}$ & $\begin{array}{l}\text { Sarajevo, Bosnia } \\
\text { and Herzegovina }\end{array}$ & $\begin{array}{l}\text { Nationality: Turkish }(58.9 \%) \text {, non-Turkish }(41.1 \%) \\
\text { Gender: male }(59 \%) \text {, female }(41 \%) \\
\text { Age: } 25 \text { and under }(10.4 \%), 25-34(25.3 \%), 35-44 \\
(29.4 \%), 45-59(15.4 \%), 60 \text { and more }(3.3 \%)\end{array}$ \\
\hline
\end{tabular}


Table 1. Cont.

\begin{tabular}{|c|c|c|c|c|c|}
\hline Article & Author, Year & Type of Study & Research Tools/Size of Sample & City/Country & Sample Population \\
\hline [84] & $\begin{array}{l}\text { Kazemi, Hesam, Rad, } \\
\quad \text { Cheraghi, } 2018\end{array}$ & Survey & 380 questionnaires & $\begin{array}{l}\text { Iranian cities: Astara, Astaneh, } \\
\text { Amlash, Bandare, Anzali, } \\
\text { Talesh, Rudhab, Rudsar, Rasht, } \\
\text { Rezvan, Shahr, Masal, Siahkal, } \\
\text { Shaft, Sowme'eh Sara, Fuman, } \\
\text { Lahijan, Langrud, }\end{array}$ & $\begin{array}{l}\text { Female tourists visiting the cities in Gilan province (according } \\
\text { to Authors' explanations: women tend to be more precise and } \\
\text { accurate than men in visual fields) }\end{array}$ \\
\hline [85] & Tasci, 2018 & Survey & $\begin{array}{l}2475 \text { questionnaires collected } \\
2318 \text { questionnaires analyzed }\end{array}$ & $\begin{array}{l}\text { New York, Orlando, Las Vegas, } \\
\text { Tampa, Miami, favorite city on } \\
\text { the US }\end{array}$ & $\begin{array}{c}\text { Nationality: US residents }(95 \%) \text {, other country residents }(5 \%) \\
\text { Age: average } 33.6 \\
\text { Gender: female }(50.3 \%) \text {, male }(49.1 \%)\end{array}$ \\
\hline [86] & $\begin{array}{l}\text { Stojanovic, Andreu, } \\
\text { Curras-Peres, } 2018\end{array}$ & Survey & 249 questionnaires & Valencia, Spain & $\begin{array}{l}\text { Nationality (place of origin): Europe (61.8\%), Australia (15.3\%), } \\
\text { S. America }(10.8 \%) \text {, N. America }(10.4 \%) \text {, Asia }(1.6 \%)\end{array}$ \\
\hline [87] & Kim, Lee, 2018 & Survey & $\begin{array}{l}385 \text { distributed questionnaires, } 350 \\
\text { selected as valid and finally used in } \\
\text { the analysis }\end{array}$ & Seoul & $\begin{array}{c}\text { Nationality: Chinese tourists } \\
\text { Gender: male }(43.4 \%) \text {, female }(56.6 \%) \\
\text { Age: } 20-29(35.1 \%), 30-39(31.1 \%), 40-49(24.9 \%) \text {, over } 50(8.9 \%)\end{array}$ \\
\hline [88] & Lee, 2017 & Survey & $\begin{array}{l}310 \text { questionnaires distributed, } \\
300 \text { questionnaires analyzed }\end{array}$ & $\begin{array}{l}\text { Beijing, Shanghai, } \\
\text { Guangzhou, Chengdu, } \\
\text { Chongqing, Wuhan, } \\
\text { Nanjing, Hangzhou, } \\
\text { and Tianjin. }\end{array}$ & $\begin{array}{c}\text { Nationality: Chinese }(64.3 \%) \text { and foreign }(35.7 \%) \text { tourists } \\
\text { Gender: male }(32 \%) \text {, female }(68 \%) \\
\text { Age: under } 20(9.0 \%), 20-29(66.7 \%), 30-39(19.3 \%) \\
\text { more than } 40(5.0 \%)\end{array}$ \\
\hline [89] & Tasseven, Ardahanlioglu, 2017 & Survey & 400 questionnaires distributed & Istanbul, Turkey & $\begin{array}{c}\text { Nationality: German }(20.5 \%) \text {, Americans }(14.3 \%) \text {, French }(9.8 \%) \text {, } \\
\text { Australian }(8.8 \%), \text { British }(5.8 \%), \text { Spanish }(5.8 \%) \text {, and others } \\
\text { Gender: male }(48.8 \%) \text {, female }(51.3 \%) \\
\text { Age: under } 18(1.0 \%), 18-27(55.3 \%), 28-38(29.8 \%) \text {, } \\
39-49(8.3 \%) \text {, over 50 }(5.8 \%)\end{array}$ \\
\hline [90] & Vinh, Nga, Nguyen, 2017 & Survey & 252 questionnaires analyzed & Danang, Vietnam & $\begin{array}{l}\text { Gender: male }(43.25 \%) \text {, female }(56.75 \%) \\
\text { Age: under } 40(38.49 \%) \text {, over } 40(61.51 \%)\end{array}$ \\
\hline [91] & Pirnar, Igneci, Tutuncuoglu, 2017 & Case study & primary and secondary research data & Izmir, Turkey & n/a \\
\hline [92] & $\begin{array}{l}\text { Esmaili, Rezaei, Abbasi, } \\
\quad \text { Eskandari, } 2017\end{array}$ & Survey & 385 questionnaires & Khalkhal City, Iran & $\begin{array}{c}\text { Gender: male }(66.4 \%) \text {, female }(33.6 \%) \\
\text { Age: } 18-25(30.5 \%), 26-35(43.5 \%), 36-45(17.9 \%) \text {, } \\
45 \text { and older }(7.8 \%)\end{array}$ \\
\hline [93] & $\begin{array}{l}\text { Alizadeh, Khamseh, } \\
\text { Ettehadi, } 2016\end{array}$ & Survey & $\begin{array}{l}242 \text { questionnaires distributed among } \\
\text { post graduated specialists and } 196 \\
\text { questionnaires analyzed }\end{array}$ & Mashhad, Iran & $\begin{array}{c}\text { Gender: male }(75.4 \%) \text {, female }(24.6 \%) \\
\text { Age: } 20-25(16.4 \%), 25-30(56.5 \%), 30-40(16.1 \%) \text {, } \\
\text { more than } 40(10.9 \%)\end{array}$ \\
\hline [94] & Salehzadeh, Pool, Soleimani, 2016 & Survey & $\begin{array}{l}442 \text { questionnaires distributed } \\
367 \text { questionnaires analyzed }\end{array}$ & Pool, Iran & $\begin{array}{l}\text { Domestic tourists, detailed data related to age and } \\
\text { gender not available }\end{array}$ \\
\hline [95] & $\begin{array}{l}\text { Peighambari, Sattari, Foster, } \\
\text { Wallström, } 2016\end{array}$ & Survey & $\begin{array}{l}6010 \text { questionnaires distributed } \\
1552 \text { questionnaires collected } \\
1451 \text { questionnaires analyzed }\end{array}$ & Lulea, Sweden & $\begin{array}{l}\text { Gender: male }(45.2 \%) \text {, female ( } 54.8 \%) \\
\text { Participants: current residents of Lulea }\end{array}$ \\
\hline
\end{tabular}


Table 1. Cont

\begin{tabular}{|c|c|c|c|c|c|}
\hline Article & Author, Year & Type of Study & Research Tools/Size of Sample & City/Country & Sample Population \\
\hline [96] & Kashif, Samsi, Sariffudin, 2015 & Survey & $\begin{array}{l}500 \text { questionnaires distributed } \\
237 \text { questionnaires analyzed }\end{array}$ & $\begin{array}{l}\text { Lahore, Pakistan } \\
\text { (Lahore Fort) }\end{array}$ & $\begin{array}{c}\text { Gender: male }(48.1 \%) \text {, female }(51.9 \%) \\
\text { Age: below } 30(43.9 \%), 31-45(39.7 \%), 45 \text { and } \\
\text { above }(16.5 \%)\end{array}$ \\
\hline [97] & Wong, Teoh, 2015 & Survey & $\begin{array}{c}1750 \text { questionnaires distributed } \\
1093 \text { questionnaires collected } \\
\text { Presumption: } 1000 \text { of international tourists } \\
\text { visited and stayed at least one night in one of } \\
\text { analyzed city (200 questionnaires per } 1 \text { city) }\end{array}$ & $\begin{array}{l}5 \text { Malaysian cities/urban } \\
\text { destinations: } \\
\text { Kuala Lumpur, Penang, Melaka, } \\
\text { Kota Kinabalu, and Kuching. }\end{array}$ & $\begin{array}{l}\text { sample drawn from tourists who had joined tour } \\
\text { programs organized by selected Malaysian inbound tour } \\
\text { operators in peninsula Malaysia and East Malaysia, and } \\
\text { had stayed at least one night as part of the tour in the } \\
\text { Malaysian urban destinations }\end{array}$ \\
\hline [98] & Jørgensen, 2014 & Case study & primary and secondary data analysis & Horsens, Denmark & $\mathrm{n} / \mathrm{a}$ \\
\hline [99] & Kladou, Kehagias, 2014 & Survey & $\begin{array}{l}401 \text { questionnaires collected, } \\
382 \text { questionnaires analyzed }\end{array}$ & Rome, Italy & $\begin{array}{c}\text { Nationality: American }(16.9 \%) \text {, German }(10.7 \%) \text {, British } \\
\text { (10.5\%), and French }(3.7 \%) \\
\text { Gender: male }(40.8 \%) \text {, female }(59.1 \%) \\
\text { Age: } 30-39(24.7 \%), 16-19(23.7 \%), 20-29(20.4 \%)\end{array}$ \\
\hline [100] & $\begin{array}{l}\text { Andéhn, Kazeminia, Lucarelli, } \\
\text { Sevin, } 2014\end{array}$ & $\begin{array}{c}\text { Twitter post } \\
\text { analysis }\end{array}$ & 73,763 tweets & Stockholm, Sweden & $\begin{array}{l}\text { Data divided into } 23 \text { segments each spanning } \\
\text { approximately } 4 \text { days }\end{array}$ \\
\hline [101] & Zenker, 2014 & $\begin{array}{l}\text { Laboratory } \\
\text { experiment }\end{array}$ & $\begin{array}{l}\text { 1st stage }-41 \text { participants, } \\
\text { 2nd stage }-20 \text { participants }\end{array}$ & Hamburg, Germany & $\begin{array}{c}\text { master students (considered to be talents) as participants } \\
\text { because of their high value as stakeholders for } \\
\text { the city of Hamburg }\end{array}$ \\
\hline [102] & $\begin{array}{l}\text { Abbasi, Roosta, } \\
\text { Nourbakhsh, } 2014\end{array}$ & Survey & 330 tourists & Shiraz, Iran & International tourists travelling to Shiraz \\
\hline [103] & Chan, 2014 & Case study & Secondary data applying to linear programming & Hong-Kong & $\mathrm{n} / \mathrm{a}$, secondary data \\
\hline [104] & Shafranskaya, Potapov, 2014 & Survey & 151 questionnaires & Perm, Russia & $\begin{array}{l}\text { gender: female ( } 57 \%) \text {, male }(43 \%) \\
\text { age: average } 32 \text { years }\end{array}$ \\
\hline [105] & Hakala, Lemmetyinen, 2013 & Case study & $\begin{array}{l}\text { Triangulation: in-depth personal interviews, } \\
\text { press articles analysis, statistical data analysis }\end{array}$ & Turku, Finland & $\begin{array}{l}\mathrm{n} / \mathrm{a} \text {, secondary (statistical data, press articles) } \\
\text { primary data (in-depth interviews) }\end{array}$ \\
\hline [106] & Yuwo, Ford, Purwanegara, 2013 & Survey & 400 questionnaires & Bandung, Indonesia & $\begin{array}{c}\text { Nationality: Indonesian }(90.7 \%) \\
\text { Gender: female }(52 \%) \text {, male }(48 \%) \\
\text { Age: } 18-24(64 \%), 25-34(24.7 \%) \text {, over } 35(11.3 \%)\end{array}$ \\
\hline [107] & $\begin{array}{l}\text { Lemmetyinen, Go, } \\
\text { Luonila, } 2013\end{array}$ & Case study & $\begin{array}{l}\text { Triangulation: secondary data analysis, content } \\
\text { analysis of national print media (top 10), content } \\
\text { analysis of national, local media and } \\
\text { international networks }\end{array}$ & Pori, Finland & $\mathrm{n} / \mathrm{a}$ \\
\hline [108] & Al-Azzam, 2013 & Survey & $\begin{array}{l}500 \text { questionnaires distributed } \\
400 \text { questionnaires collected } \\
350 \text { questionnaires analyzed }\end{array}$ & $\begin{array}{l}3 \text { cities in Jordan: Aqaba, } \\
\text { Amman, Ajloun }\end{array}$ & $\begin{array}{l}\text { Gender: female (57.5\%), male (42.5\%) } \\
\text { Age: } 18-25(15.3 \%), 26-45(24.2 \%), 46-60(18.9 \%)\end{array}$ \\
\hline [109] & Florek, 2012 & CATI & 800 telephone interviews & $\begin{array}{l}\text { Poznań, Poland } \\
\text { Wrocław, Poland }\end{array}$ & $\begin{array}{l}\text { national sample reflecting the Polish population's } \\
\text { distribution with respect to gender, age, education and } \\
\text { place of residence }\end{array}$ \\
\hline [110] & Boo, Busser, Baloglu, 2009 & Survey & $\begin{array}{c}\text { 10,000 email requests to participate in the survey } \\
\text { send, response rate } 5.1 \% \text { (Las Vegas }=270 \\
\text { Atlantic City }=240)\end{array}$ & $\begin{array}{l}\text { Las Vegas, US } \\
\text { Atlantic City, US }\end{array}$ & $\begin{array}{l}\text { Las Vegas: Caucasian }(89 \%) \text {, male }(34 \%) \text {, female }(66 \%) \\
\text { Atlantic City: Caucasian }(90 \%) \text {, male }(35 \%) \text {, female }(65 \%)\end{array}$ \\
\hline
\end{tabular}


Table 2. Research specifications for the studies included in the SLR.

\begin{tabular}{|c|c|c|c|c|}
\hline Article & Factor/Variable & Hypotheses or Research Questions & Measurement Items & Data Analysis \\
\hline [75] & $\begin{array}{c}\text { customer-based destination BE: destination image (DI), destination awareness } \\
\text { (DA), perceived destination quality (PDQ), destination loyalty (DL) } \\
\text { DMO-generated social media communication (DMO-SMC) } \\
\text { tourist-generated social media communication (T-SMC) } \\
\text { brand engagement with destination (DBE) }\end{array}$ & $\begin{array}{c}\text { DMO-SMC } \rightarrow \text { DA and DI }(+) \\
\text { T-SMC } \rightarrow \text { DA and DI (+) } \\
\text { DA } \rightarrow \text { PDQ }(+) \\
\text { DI } \rightarrow \text { PDQ }(+) \\
\text { PDQ } \rightarrow \text { DL }(+)\end{array}$ & $\begin{array}{c}\text { 7-point Likert-type scale: } \\
\text { DMO-SMC-4 items } \\
\text { T-SMC-4 items } \\
\text { DA-3 items, PDQ-3 items } \\
\text { DI-4 items, DL-4 items } \\
\text { DBE-6 items }\end{array}$ & $\begin{array}{l}\text { CFA } \\
\text { SEM }\end{array}$ \\
\hline [76] & $\begin{array}{c}\text { destination brand awareness (DBA) } \\
\text { destination service quality (DSQ) } \\
\text { destination natural quality (DNQ) } \\
\text { destination functional value (DFV) } \\
\text { destination hedonic value (DHV) } \\
\text { destination brand trust (DBT) } \\
\text { destination brand satisfaction (DBS) } \\
\text { destination brand loyalty (DBL) }\end{array}$ & $\begin{aligned} \mathrm{DBA} \rightarrow \mathrm{DSQ}(+), \mathrm{DBA} \rightarrow \mathrm{DNQ}(+) \\
\mathrm{DSQ} \rightarrow \mathrm{DFV}(+), \mathrm{DSQ} \rightarrow \mathrm{DHV}(+) \\
\mathrm{DNQ} \rightarrow \mathrm{DFV}(+), \mathrm{DNQ} \rightarrow \mathrm{DHV}(+) \\
\mathrm{DFV} \rightarrow \mathrm{DBT}(+) \\
\text { DHV } \rightarrow \text { DBT }(+) \\
\text { DBT } \rightarrow \text { DBS }(+) \\
\text { DBS } \rightarrow \text { DBL }(+)\end{aligned}$ & $\begin{array}{l}\text { DQS-23 items } \\
\text { DNQ-5 items } \\
\text { DFV- } 8 \text { items } \\
\text { DHV-10 items } \\
\text { DBT- } 4 \text { items } \\
\text { DBS-3 items } \\
\text { DBL- } 3 \text { items }\end{array}$ & $\begin{array}{l}\text { CFA } \\
\text { SEM }\end{array}$ \\
\hline [77] & $\begin{array}{l}\text { overall destination brand equity (ODBE) } \\
\text { destination brand awareness (DBA) } \\
\text { destination perceived quality (DPQ) } \\
\text { destination brand image (DBI) } \\
\text { destination brand loyalty (DBL) }\end{array}$ & $\begin{array}{c}\mathrm{DBA} \rightarrow \mathrm{DBI}(+), \mathrm{DBA} \rightarrow \mathrm{DPQ}(+) \\
\mathrm{DBI} \rightarrow \mathrm{DPQ}(+), \mathrm{DBI} \rightarrow \mathrm{DBL}(+) \\
\mathrm{DBQ} \rightarrow \mathrm{DBL}(+) \\
\mathrm{DBA} \rightarrow \mathrm{DBL}(+), \mathrm{DBA} \rightarrow \mathrm{ODBE}(+) \\
\mathrm{DBI} \rightarrow \mathrm{ODBE}(-) \\
\mathrm{DPQ} \rightarrow \mathrm{ODBE}(+), \mathrm{DBL} \rightarrow \mathrm{ODBE}(+)\end{array}$ & $\begin{array}{l}\text { DBA-4 items } \\
\text { DBI-4 items } \\
\text { DPQ-4 items } \\
\text { DBL-3 items } \\
\text { ODBE-3 items }\end{array}$ & $\begin{array}{l}\text { CFA } \\
\text { SEM }\end{array}$ \\
\hline [78] & $\begin{array}{l}\text { unique selling proposition (USP) } \\
\text { brand communication (BC) } \\
\text { city branding (CB) } \\
\text { brand equity (BE) }\end{array}$ & $\begin{aligned} \mathrm{USP} & \rightarrow \mathrm{BC}, \mathrm{USP} \rightarrow \mathrm{CB} \\
\mathrm{BC} & \rightarrow \mathrm{CB} \\
\mathrm{USP} & \rightarrow \mathrm{BE}, \mathrm{BC} \rightarrow \mathrm{BE}, \mathrm{CB} \rightarrow \mathrm{BE}\end{aligned}$ & $\begin{array}{l}\text { 10-point scale: } \\
\text { USP-5 items } \\
\text { BC—5 items } \\
\text { CB-6 items } \\
\text { BE-2 items }\end{array}$ & $\begin{array}{l}\text { CFA } \\
\text { SEM }\end{array}$ \\
\hline [79] & $\begin{array}{l}\text { customer-based brand equity (CBBE) } \\
\text { brand awareness (BA) } \\
\text { brand image (BI) } \\
\text { perceived brand quality (PBQ) } \\
\text { brand loyalty (BL) }\end{array}$ & $\begin{aligned} & \mathrm{BA} \rightarrow \mathrm{CBBE}(+) \\
& \mathrm{BI} \rightarrow \mathrm{CBBE}(+) \\
& \mathrm{PBQ} \rightarrow \mathrm{CBBE}(+) \\
& \mathrm{BL} \rightarrow \mathrm{CBBE}(+)\end{aligned}$ & $\begin{array}{l}\text { 5-point Likert scale to evaluate items of BA, } \\
\text { BI, PBQ, and BL } \\
\text { CBBE index of Tourism Brand calculated as } \\
\text { aggregate value of CBBE index by factor } \\
\text { weight and factor percentage }\end{array}$ & $\begin{array}{l}\text { CFA } \\
\text { SEM }\end{array}$ \\
\hline [80] & $\begin{array}{l}\text { destination brand awareness (DBA) } \\
\text { destination brand value (DBV) } \\
\text { destination brand image (DBI) } \\
\text { destination brand quality (DBQ) } \\
\text { destination brand loyalty (DBL) } \\
\text { destination brand satisfaction (DBS) }\end{array}$ & $\begin{aligned} \mathrm{DBA} \rightarrow & \mathrm{DBI}(+), \mathrm{DBA} \rightarrow \mathrm{DBV}(+) \\
\mathrm{DBI} \rightarrow & \mathrm{DBQ}(-), \mathrm{DBI} \rightarrow \mathrm{DBL}(+) \\
\mathrm{DBQ} \rightarrow & \mathrm{DBV}(+), \mathrm{DBQ} \rightarrow \mathrm{DBL}(+) \\
& \mathrm{DBV} \rightarrow \mathrm{DBS}(+) \\
& \mathrm{DBV} \rightarrow \mathrm{DBL}(+) \\
& \mathrm{DBS} \rightarrow \mathrm{DBL}(+)\end{aligned}$ & $\begin{array}{l}\text { 5-point Likert scale } \\
\text { DBA-3 items, DBI-4 items } \\
\text { DBQ-3 items, DBV-4 items } \\
\text { DBS-4 items } \\
\text { DBL-3 items }\end{array}$ & $\begin{array}{l}\text { CFA } \\
\text { SEM }\end{array}$ \\
\hline [81] & $\begin{array}{c}\text { city brand equity (CBE) } \\
\text { brand awareness (BA) } \\
\text { perceived quality (PQ) } \\
\text { attitude toward the brand (AB) } \\
\text { brand loyalty (BL) } \\
\text { brand image (BI) } \\
\text { city brand preference (CBP) } \\
\text { city brand index (CBI) }\end{array}$ & $\begin{aligned} & \mathrm{BA} \rightarrow \mathrm{CBE}(+) \\
& \mathrm{PQ} \rightarrow \mathrm{CBE}(+) \\
& \mathrm{BL} \rightarrow \mathrm{CBE}(-) \\
& \mathrm{AB} \rightarrow \mathrm{CBE}(-) \\
& \mathrm{AB} \rightarrow \mathrm{BI}(+) \\
& \mathrm{BI} \rightarrow \mathrm{CBE}(+) \\
& \mathrm{CBE} \rightarrow \mathrm{CBP}(+)\end{aligned}$ & $\begin{array}{c}11 \text { point Likert scale } \\
\text { AB-5 items } \\
\text { BI-3 items } \\
\text { BA-3 items } \\
\text { BL-3 items } \\
\text { PQ-3 items } \\
\text { CBE-2 items } \\
\text { CBP-3 items }\end{array}$ & 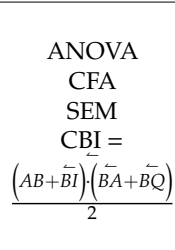 \\
\hline
\end{tabular}


Table 2. Cont.

\begin{tabular}{|c|c|c|c|c|}
\hline Article & Factor/Variable & Hypotheses or Research Questions & Measurement Items & Data Analysis \\
\hline [82] & $\begin{array}{l}\text { destination brand awareness (DBA) } \\
\text { destination brand image (DBI) } \\
\text { destination brand quality (DBQ) } \\
\text { destination brand value (DBV) } \\
\text { destination brand loyalty (DBL) }\end{array}$ & $\begin{array}{c}\mathrm{DBA} \rightarrow \mathrm{DBL}(+) \\
\mathrm{DBI} \rightarrow \mathrm{DBL}(+) \\
\mathrm{DBQ} \rightarrow \mathrm{DBL}(\mathrm{A}+, \mathrm{F}-) \\
\mathrm{DBV} \rightarrow \mathrm{DBL}(+)\end{array}$ & $\begin{array}{l}\text { 7-point Likert-type scale } \\
\text { DBA-4 items, DBI-4 items } \\
\text { DBQ-4 items, DBV—4 items } \\
\text { DBL-3 items }\end{array}$ & $\begin{array}{l}\text { CFA } \\
\text { SEM }\end{array}$ \\
\hline [83] & $\begin{array}{c}\text { destination image (DI) } \\
\text { brand performance (quality of experience) BP } \\
\text { customer feelings about the vacation experience (CF), customer judgments (CJ) } \\
\text { customer brand resonance (CBR): behavioral loyalty (BL), attitudinal } \\
\text { attachment (AT) }\end{array}$ & $\begin{aligned} \mathrm{BP} & \rightarrow \mathrm{CJ}(+) \\
\mathrm{BP} & \rightarrow \mathrm{CF}(+) \\
\mathrm{CF} & \rightarrow \mathrm{CBR}(+) \\
\mathrm{CJ} & \rightarrow \mathrm{CBR}(+)\end{aligned}$ & $\begin{array}{l}\text { 5-point Likert-type scale } \\
\text { DI-8 items } \\
\text { BP-7 items, CF-4 items } \\
\text { CJ-4 items, BL-2 items } \\
\text { AT- } 3 \text { items }\end{array}$ & $\begin{array}{l}\text { EFA } \\
\text { SEM }\end{array}$ \\
\hline [84] & $\begin{array}{c}\text { tourism destination brand equity } \\
\text { brand image (BI) } \\
\text { perceived quality (PQ) } \\
\text { brand awareness (BA) }\end{array}$ & $\begin{array}{l}\text { BI, PQ, BA I BL as elements of BE } \\
\text { Clusters of BE (week, average, great) }\end{array}$ & $\begin{array}{l}\text { 5-point Likert-type scale } \\
\text { BI-13 items } \\
\text { PQ-12 items, BA—4 items } \\
\text { BL-4 items }\end{array}$ & Cluster analysis \\
\hline [85] & $\begin{array}{c}\text { consumer-based Brand Equity (CBBE): } \\
\text { familiarity (BF), image (BI) } \\
\text { consumer value: perception of value for money (CV); brand value: perception } \\
\text { of price premium (BV) }\end{array}$ & $\begin{aligned} & \mathrm{BF} \rightarrow \mathrm{BI}(+) \\
& \mathrm{BF} \rightarrow \mathrm{CV}(+), \mathrm{BF} \rightarrow \mathrm{BL}(+) \\
& \mathrm{BI} \rightarrow \mathrm{CV}(+) \\
& \mathrm{BI} \rightarrow \mathrm{BV}(+), \mathrm{BI} \rightarrow \mathrm{BL}(+) \\
& \mathrm{BV} \rightarrow \mathrm{CV}(+), \mathrm{CV}-\mathrm{BL}(+)\end{aligned}$ & 10-point single-item scales & $\begin{array}{l}\text { CFA } \\
\text { SEM }\end{array}$ \\
\hline [86] & $\begin{array}{l}\text { communication source: social media use (SM) } \\
\text { Brand equity (BE): } \\
\text { brand identification as brand awareness (BA) } \\
\text { responses to the brand as brand quality (BQ) and customer value }(\mathrm{CV}) \\
\text { brand meaning as brand image (BI) affective and cognitive } \\
\text { Relationships with the brand as WOM communication }\end{array}$ & $\begin{array}{c}\mathrm{SM} \rightarrow \mathrm{BA}(+) \\
\mathrm{BA} \rightarrow \mathrm{BI} \text { affective and cognitive }(+) \\
\mathrm{BA} \rightarrow \mathrm{BQ}(+), \mathrm{BA} \rightarrow \mathrm{CV}(+) \\
\mathrm{BA} \rightarrow \text { WOM }(-) \text { and eWOM }(+) \\
\mathrm{BI} \text { affective and cognitive } \rightarrow \text { WOM }(-) \text { and eWOM }(-) \\
\mathrm{BQ} \rightarrow \text { WOM }(+) \text { and eWOM }(+) \\
\mathrm{CV} \rightarrow \text { WOM }(+) \text { and eWOM }(+)\end{array}$ & $\begin{array}{l}\text { differential scale of seven points } \\
\text { SM-3 items, BA-4 items } \\
\text { BI: cultural image- } 4 \text { items } \\
\text { BI: nature- }-3 \text { items } \\
\text { BI: attractions and leisure }-4 \text { items } \\
\text { BI: Mediterranean city }-2 \text { items } \\
\text { BI: affective }-4 \text { items } \\
\text { BQ-5 items, CV-3 items } \\
\text { WOM-3 items, eWOM-3 items }\end{array}$ & $\begin{array}{l}\text { CFA } \\
\text { SEM }\end{array}$ \\
\hline [87] & $\begin{array}{c}\text { tourism destination influencing factors (TDIF): price (p), advertisement (ad), } \\
\text { promotion (pm), tradition (tr) } \\
\text { brand perceived quality (BPQ) } \\
\text { brand awareness (BA) } \\
\text { brand image (BI) } \\
\text { brand loyalty (BL) }\end{array}$ & $\begin{array}{c}\text { TDIF: } \mathrm{p}(+), \mathrm{ad}(-), \mathrm{pm}(-), \operatorname{tr}(+) \rightarrow \mathrm{BPQ} \\
\text { TDIF } \mathrm{p}(+), \mathrm{ad}(+), \mathrm{pm}(-), \operatorname{tr}(+) \rightarrow \mathrm{BA} \\
\text { TDIF } \mathrm{p}(-), \mathrm{ad}(+), \mathrm{pm}(-), \operatorname{tr}(-) \rightarrow \mathrm{BI} \\
\mathrm{BA} \rightarrow \mathrm{BPQ}(-), \mathrm{BA} \rightarrow \mathrm{BI}(+) \\
\mathrm{BPQ} \rightarrow \mathrm{BI}(+) \\
\mathrm{BI} \rightarrow \mathrm{BL}(+), \mathrm{BPQ} \rightarrow \mathrm{BL}(+)\end{array}$ & $\begin{array}{c}\text { TDIF-14 items } \\
\text { BPQ, BA, BI-11 items }\end{array}$ & $\begin{array}{l}\text { CFA } \\
\text { SEM }\end{array}$ \\
\hline [88] & $\begin{array}{l}\text { brand awareness (BA) } \\
\text { brand identity (BId) } \\
\text { brand image (BI) } \\
\text { perceived quality (PQ) } \\
\text { brand loyalty (BL) }\end{array}$ & $\begin{array}{l}\mathrm{BA} \rightarrow \mathrm{BId}(-), \mathrm{BI} \rightarrow \mathrm{BId}(+) \\
\mathrm{PQ} \rightarrow(\mathrm{BId})(+), \mathrm{BId} \rightarrow \mathrm{BL}(+) \\
\mathrm{BId} \text { mediates BA and BL }(+) \\
\text { BId mediates BI and BL }(+) \\
\text { BId mediates PQ and BL }(+)\end{array}$ & $\begin{array}{l}\text { 5-point Likert-type scale } \\
\text { BA-6 items, BI-3 items } \\
\text { PQ-6 items, BL-3 items } \\
\text { BId-5 items }\end{array}$ & $\begin{array}{l}\text { CFA } \\
\text { SEM }\end{array}$ \\
\hline [89] & $\begin{array}{l}\text { enduring travel involvement (ETI) } \\
\text { destination brand equity (DBE) elements: cultural assets (CA), brand } \\
\text { awareness (BA), brand associations (BAs), perceived quality (PQ), brand } \\
\text { loyalty (BL), overall brand equity (BE) } \\
\text { consumer satisfaction (CS), future behavior (FB) }\end{array}$ & $\begin{aligned} \mathrm{ETI} & \rightarrow \mathrm{DBE} \\
\mathrm{DBE} & \rightarrow \mathrm{BE}(+) \\
\mathrm{DBE} & \rightarrow \mathrm{CS}(+) \\
\mathrm{DBE} & \rightarrow \mathrm{FB}(+) \\
\mathrm{BE} & \rightarrow \mathrm{CS}(+) \\
\mathrm{BE} & \rightarrow \mathrm{FB}(+)\end{aligned}$ & $\begin{array}{l}\text { ETI-5 items (7-point scale) } \\
\text { 5-point Likert-type scale } \\
\text { CA-10 items } \\
\text { BA-4 items } \\
\text { Bas-9 items } \\
\text { PQ-4 items }\end{array}$ & $\begin{array}{l}\text { CFA } \\
\text { SEM }\end{array}$ \\
\hline
\end{tabular}


Table 2. Cont.

\begin{tabular}{|c|c|c|c|c|}
\hline Article & Factor/Variable & Hypotheses or Research Questions & Measurement Items & Data Analysis \\
\hline [90] & $\begin{array}{l}\text { destination brand equity (DBE) } \\
\text { destination brand awareness (DBA) } \\
\text { destination perceived quality (DPQ) } \\
\text { destination brand image (DBI) } \\
\text { destination brand loyalty (DBL) }\end{array}$ & $\begin{aligned} \mathrm{DBA} & \rightarrow \mathrm{DBI}(+) \\
\mathrm{DBA} & \rightarrow \mathrm{DPQ}(-) \\
\mathrm{DBA} & \rightarrow \text { DBL }(-) \\
\mathrm{DBI} & \rightarrow \text { DPQ }(+) \\
\mathrm{DBI} & \rightarrow \text { DBL }(+) \\
\mathrm{DBQ} & \rightarrow \text { DBL }(+)\end{aligned}$ & $\begin{array}{l}\text { DBA-4 items } \\
\text { DBI-4 items } \\
\text { DPQ-4 items } \\
\text { DBL-4 items }\end{array}$ & $\begin{array}{l}\text { CFA } \\
\text { SEM }\end{array}$ \\
\hline [91] & $\begin{array}{l}\text { place brand equity: } \\
\text { familiarity of city attractions } \\
\text { favorability } \\
\text { uniqueness }\end{array}$ & $\begin{array}{l}\text { Elements of place brand equity (PBE) as strengths, } \\
\text { weaknesses, opportunities, and threats in SWOT (Strengths, } \\
\text { Weaknesses, Opportunities and Threats) analysis }\end{array}$ & $\begin{array}{l}\text { Elements of SWOT analysis } \\
\text { strengths: } 18 \text { items } \\
\text { weaknesses: } 20 \text { items } \\
\text { opportunities: } 8 \text { items } \\
\text { threats: } 7 \text { items }\end{array}$ & SWOT analysis \\
\hline [92] & $\begin{array}{c}\text { destination brand loyalty (DBL) } \\
\text { destination brand equity (DBE) } \\
\text { destination perceived quality (DPQ) } \\
\text { destination brand image (DBI) } \\
\text { destination perceived value (DBV) } \\
\text { price (P), distribution (D), sale promotion (SP), } \\
\text { physical evidence (PE), people (Pe) }\end{array}$ & $\begin{aligned} & \mathrm{P} \rightarrow \mathrm{DPQ}(-) \text { and DBI }(+) \text { and DBL }(+) \\
& \mathrm{PE} \rightarrow \mathrm{DPQ}(+) \text { and DPV }(+) \text { and DBL }(+) \\
& \mathrm{D} \rightarrow \mathrm{DPQ}(+) \text { and DBI }(+) \text { and DPV }(+) \text { and DBL }(+) \\
& \mathrm{Pe} \rightarrow \mathrm{DPQ}(+) \text { and DPV }(+) \text { and DBL }(-) \\
& \mathrm{SP} \rightarrow \mathrm{DPQ}(+) \text { and DBI }(+) \text { and DPV }(+) \text { and DBL }(-) \\
& \mathrm{DPQ} \rightarrow \mathrm{DBL}(+), \mathrm{DBI} \rightarrow \mathrm{DBL}(+), \mathrm{DBV} \rightarrow \mathrm{DBL}(+)\end{aligned}$ & $\begin{array}{l}\text { 5-point Likert-type scale } \\
\text { P-3 items, D-3 items } \\
\text { Pe-3 items, SP-5 items } \\
\text { DPQ-4 items, DPV-3 items } \\
\text { DBI-6 items, DBL-4 items }\end{array}$ & CFA \\
\hline [93] & $\begin{array}{c}\text { city brand equity (CBE): brand awareness, perceived quality of brand } \\
\text { Brand identity (BId) } \\
\text { Expert's immigration (EI) }\end{array}$ & $\begin{aligned} \mathrm{CBE} & \rightarrow \mathrm{BId}(+) \\
\mathrm{BId} & \rightarrow \mathrm{EI}(+) \\
\mathrm{CBE} & \rightarrow \mathrm{EI}(+)\end{aligned}$ & $\begin{array}{l}\text { 5-point Likert-type scale } \\
\text { CBE-14 items } \\
\text { BId—6 items }\end{array}$ & SEM \\
\hline [94] & $\begin{array}{l}\text { brand personality (BP) } \\
\text { brand equity (BE) } \\
\text { revisit intention (RI) }\end{array}$ & $\begin{array}{l}\mathrm{BP} \rightarrow \mathrm{BE}(+) \\
\mathrm{BE} \rightarrow \mathrm{RI}(+) \\
\mathrm{BP} \rightarrow \mathrm{RI}(+)\end{array}$ & $\begin{array}{l}\mathrm{BE}-12 \text { items } \\
\mathrm{RI}-4 \text { items }\end{array}$ & $\begin{array}{l}\text { CFA } \\
\text { SEM }\end{array}$ \\
\hline [95] & $\begin{array}{l}\text { place brand equity (PBE): } \\
\text { brand identity (BId) } \\
\text { brand image (BI) } \\
\text { brand loyalty (BL) } \\
\text { brand personality (BP) }\end{array}$ & $\begin{array}{c}\text { difference between how BId from city officials' perspective } \\
\text { and BI from city residents' perspective are related to BL } \\
\text { toward a city }(+) \\
\text { difference between BL toward a city among city officials and } \\
\text { city residents }(+) \\
\text { difference between how city officials and city residents } \\
\text { perceive the BP of that city }(+)\end{array}$ & $\begin{array}{l}\text { 5-point Likert-type scale } \\
\text { BId-5 items } \\
\text { BI-5 items } \\
\text { BL-3 items } \\
\text { BP-5 items }\end{array}$ & $\begin{array}{l}\text { CFA } \\
\text { SEM }\end{array}$ \\
\hline [96] & $\begin{array}{c}\text { brand equity (BE) } \\
\text { brand awareness (BA) } \\
\text { brand image (BI): brand attitude and brand quality } \\
\text { Brand associations (Bas) } \\
\text { brand loyalty (BL) }\end{array}$ & $\begin{aligned} \mathrm{BA} \rightarrow \mathrm{BL}(-), \mathrm{BI} \rightarrow \mathrm{BL}(+) \\
\mathrm{BAs} \rightarrow \mathrm{BL}(+) \\
\mathrm{BA} \rightarrow \mathrm{BE} \mathrm{(-),} \mathrm{BI} \rightarrow \mathrm{BE}(+) \\
\mathrm{BAs} \rightarrow \mathrm{BE}(+) \\
\quad \mathrm{BL} \rightarrow \mathrm{BE}(+)\end{aligned}$ & $\begin{array}{l}\text { 5-point Likert-type scale } \\
\text { BA-3 items } \\
\text { BI-20 items } \\
\text { BAs-4 items } \\
\text { BL-4 items }\end{array}$ & $\begin{array}{l}\text { CFA } \\
\text { SEM }\end{array}$ \\
\hline [97] & $\begin{array}{c}\text { consumer based brand equity (CBBE): } \\
\text { brand performance, brand judgment } \\
\text { brand feeling } \\
\text { brand resonance, brand imagery } \\
\text { brand experience } \\
\text { destination competitiveness: } \\
\text { functional attributes, abstract attributes }\end{array}$ & $\begin{array}{l}\text { The functional attributes component of destination } \\
\text { competitiveness is the antecedent of CBBE }(+) \\
\text { There is a positive relationship between the functional } \\
\text { attributes of destination competitiveness and CBBE }(+)\end{array}$ & $\begin{array}{l}\text { 5-point Likert scale } \\
\text { Total number: } 100 \text { items related to } 6 \\
\text { elements of CBBE } \\
\text { and attitude: functional and psychological } \\
\text { number of attitudes: } 36\end{array}$ & $\begin{array}{l}\text { CFA } \\
\text { SEM }\end{array}$ \\
\hline
\end{tabular}


Table 2. Cont.

\begin{tabular}{|c|c|c|c|c|}
\hline Article & Factor/Variable & Hypotheses or Research Questions & Measurement Items & Data Analysis \\
\hline [98] & $\begin{array}{l}\text { brand equity (BE): } \\
\text { investments-shareholder perspective (In) } \\
\text { return on investment: } \\
\text { (1) shareholder and stakeholder perspective: first-order effects (RoI) } \\
\text { (2) second-order effects: impacts: intangible assets (IA) within and outside } \\
\text { the city, tangible assets (TA) within and outside the city }\end{array}$ & $\begin{array}{l}\text { In } \rightarrow \text { BE } \\
\text { RoI } \rightarrow \text { BE } \\
\mathrm{IA} \rightarrow \mathrm{BE} \\
\mathrm{TA} \rightarrow \mathrm{BE}\end{array}$ & $\begin{array}{l}\text { In-7 items } \\
\text { RoI-5 items } \\
\text { IA-10 items } \\
\text { TA-8 items }\end{array}$ & $\begin{array}{l}\text { quantitative and } \\
\text { qualitative analysis } \\
\text { of data }\end{array}$ \\
\hline [99] & $\begin{array}{l}\text { cultural destination brand equity (CDBE): cultural destination brand assets } \\
\text { (CDBA), cultural destination brand awareness (CDBAW), cultural } \\
\text { destination brand associations (CDBAs), cultural destination brand quality } \\
\text { (CDBQ), cultural destination brand loyalty (CDBL) }\end{array}$ & $\begin{aligned} & \mathrm{CDBA} \rightarrow \mathrm{CDBE}(+) \\
\mathrm{CDBAW} & \rightarrow \mathrm{CDBE}(+), \mathrm{CDBAs} \rightarrow \mathrm{CDBE}(+) \\
\mathrm{CDBQ} & \rightarrow \mathrm{CDBE}(+), \mathrm{CDBL} \rightarrow \mathrm{CDBE}(+) \\
& \mathrm{CDBA} \rightarrow \mathrm{CDBAW}(+) \\
\mathrm{CDBAW} & \rightarrow \mathrm{CDBAs}(-) \text { and } \mathrm{CDBQ}(-) \\
\mathrm{CDBAs} & \rightarrow \mathrm{CDBL}(+), \mathrm{CDBQ} \rightarrow \mathrm{CDBL}(+)\end{aligned}$ & $\begin{array}{l}\text { 5-point Likert-type scale } \\
\text { CDBA-10 items } \\
\text { CDBAW-4 items } \\
\text { CDBAs—-9 items } \\
\text { CDBQ-4 items } \\
\text { CDBL-items }\end{array}$ & $\begin{array}{l}\text { CFA } \\
\text { SEM }\end{array}$ \\
\hline [100] & $\begin{array}{l}\text { place brand equity } \\
\text { brand image } \\
\text { brand associations }\end{array}$ & $\begin{array}{l}\text { How social media could be conceptualized as a new venue for } \\
\text { place brand meaning formation? } \\
\text { How user-generated content pertains to the issue of place BE? }\end{array}$ & $\begin{array}{l}21 \text { concepts separated into three categories } \\
\text { of place brand associations: events, } \\
\text { city/country name and people }\end{array}$ & $\begin{array}{l}\text { semantic analysis } \\
\text { content analysis }\end{array}$ \\
\hline [101] & $\begin{array}{c}\text { place brand equity (PBE) } \\
\text { advanced brand concept map (aBCM): brand association } \\
\text { brand association network value (BANV): } \\
\text { favorability, strength, uniqueness of brand associations }\end{array}$ & $\begin{array}{l}\mathrm{aBCM} \rightarrow \mathrm{PBE} \\
\mathrm{BANV} \rightarrow \mathrm{PBE}\end{array}$ & $\begin{array}{l}\text { All elements evaluated on 7-point } \\
\text { Likert scale }\end{array}$ & $\begin{array}{l}\text { Advanced Brand } \\
\text { Concept Map } \\
\text { (aBCM) method }\end{array}$ \\
\hline [102] & $\begin{array}{c}\text { customer-based brand equity } \\
\text { destination brand awareness (DBA) } \\
\text { destination brand value (DBV) } \\
\text { destination brand image (DBI) } \\
\text { destination brand quality (DBQ) } \\
\text { destination brand loyalty (DBL) } \\
\end{array}$ & $\begin{array}{l}\mathrm{DBA} \rightarrow \mathrm{DBV}(-) \\
\mathrm{DBI} \rightarrow \mathrm{DBV}(+) \\
\mathrm{DBI} \rightarrow \mathrm{DBL}(+) \\
\mathrm{DBQ} \rightarrow \mathrm{DBV}(+) \\
\mathrm{DBQ} \rightarrow \mathrm{DBL}(+) \\
\mathrm{DBV} \rightarrow \mathrm{DBL}(+)\end{array}$ & $\begin{array}{l}\text { 5-point scale: } \\
\text { DBA-5 items } \\
\text { DBI-4 items } \\
\text { DBQ-3 items } \\
\text { DBV-4 items } \\
\text { DBL-6 items } \\
\end{array}$ & $\begin{array}{l}\text { CFA } \\
\text { SEM }\end{array}$ \\
\hline [103] & $\begin{array}{l}\text { groups of targeted consumers: } \\
\text { international tourist, investors, professional group, local residents } \\
\text { marketing and administrative expenditure } \\
\text { city brand equity }\end{array}$ & $\begin{array}{l}\text { How should resources be best allocated among the four key } \\
\text { target groups with the objective of maximizing CBE? } \\
\text { Elements: } \\
\text { budget constraint: marketing expenditure } \\
\text { policy constraint: governmental expenditure for marketing } \\
\text { administrative constraint: total administrative cost } \\
\text { non-negativity constraint }\end{array}$ & $\begin{array}{c}\text { Marketing expenditures spent on } 4 \\
\text { targeted groups of consumers } \\
\text { city brand equity generated by one unit of } \\
\text { marketing expenditures spent on } 4 \text { targeted } \\
\text { groups of consumers } \\
\text { total administrative cost induced by all } \\
\text { marketing activities and on } 4 \text { targeted } \\
\text { groups of consumers }\end{array}$ & Linear programming \\
\hline [104] & $\begin{array}{c}\text { city diversity }(\mathrm{CD}) \\
\text { city safety and comfort }(\mathrm{CSC}) \\
\text { professional and job changes }(\mathrm{PJ}) \\
\text { city facilities }(\mathrm{CF})\end{array}$ & $\begin{array}{l}\text { How important are city attributes in the CBE of Perm? } \\
\text { What is the structure of city utilities? }\end{array}$ & $\begin{array}{l}\text { CD-4 items } \\
\text { CSC-4 items } \\
\text { PJ-4 items } \\
\text { CF-4 items }\end{array}$ & $\begin{array}{l}\text { Conjoint analysis } \\
\text { Linear regression }\end{array}$ \\
\hline [105] & $\begin{array}{c}\begin{array}{c}\text { European Capital of Culture } \\
\text { city brand equity }\end{array} \\
\text { direct impacts (e.g., investments, purchases of services and commodities, } \\
\text { and increased tourism income) } \\
\text { Indirect or multiplier effects (the intermediate product input from partner } \\
\text { and other companies and industries, satisfying the increasing demand for } \\
\text { goods and services; new jobs created as a result of increased production) }\end{array}$ & $\begin{array}{l}\text { How can a cultural capital organization build brand } \\
\text { awareness for the year? } \\
\text { What kind of associations can and should be emphasized? } \\
\text { What can be done in order to increase positive } \\
\text { brand experiences? } \\
\text { How can the organization ensure brand loyalty? }\end{array}$ & $\begin{array}{l}\text { qualitative analysis of interviews } \\
\text { and press articles } \\
\text { quantitative analysis of statistical data }\end{array}$ & $\begin{array}{l}\text { content analysis } \\
\text { data analysis }\end{array}$ \\
\hline
\end{tabular}


Table 2. Cont.

\begin{tabular}{|c|c|c|c|c|}
\hline Article & Factor/Variable & Hypotheses or Research Questions & Measurement Items & Data Analysis \\
\hline [106] & $\begin{array}{l}\text { customer-based brand equity for a tourism destination (CBBETD): } \\
\text { awareness, image, quality, loyalty, }\end{array}$ & $\begin{array}{l}\text { Can the CBBETD scale be adapted for the city of Bandung? } \\
\text { Which of the sub-dimensions of CBBETD will be useful for strategic } \\
\text { decisions regarding tourism in the city of Bandung? }\end{array}$ & $\begin{array}{l}\text { DA-5 items } \\
\text { DI-17 items } \\
\text { DQ-12 items } \\
\text { DL-7 items }\end{array}$ & CFA \\
\hline [107] & $\begin{array}{l}\text { place brand equity } \\
\text { destination awareness } \\
\text { perceived quality } \\
\text { brand associations } \\
\text { destination loyalty } \\
\text { destination positioning } \\
\text { special event/jazz festival }\end{array}$ & $\begin{array}{l}\text { What is the potential for image transfer between the special event } \\
\text { and the host city? } \\
\text { What are the event's contributions to destination awareness, } \\
\text { perceived quality, brand associations, and destination } \\
\text { loyalty and positioning? } \\
\text { How can cultural producers leverage the distinct reputation of an } \\
\text { event (herein Pori Jazz) as a magnet attracting resources to the host } \\
\text { city (Pori in this case) (on the local and the global scale? }\end{array}$ & $\begin{array}{l}\text { 'inside-in' strategic perspective - secondary } \\
\text { data on local strategies } \\
\text { 'inside-out' strategic perspective on the } \\
\text { national print media by means of } \\
\text { content analysis } \\
\text { 'outside-in's strategic perspective on Pori's } \\
\text { international reputation in a content analysis } \\
\text { of national and local media reflections on the } \\
\text { international networks and connections the } \\
\text { jazz organization }\end{array}$ & Content analysis \\
\hline [108] & $\begin{array}{l}\text { destination brand awareness (DBA) } \\
\text { destination brand image (DBI) } \\
\text { destination brand salience (DBS) } \\
\text { destination satisfaction (DS) } \\
\text { destination brand loyalty (DBL) } \\
\end{array}$ & $\begin{aligned} & \mathrm{DBA} \rightarrow \mathrm{DBL}(+) \\
& \mathrm{DBI} \rightarrow \text { DBL }(+) \\
& \mathrm{DBS} \rightarrow \mathrm{DBL}(+) \\
& \mathrm{DS} \rightarrow \mathrm{DBL}(+)\end{aligned}$ & $\begin{array}{l}\text { DBA-6 items } \\
\text { DBI-5 items } \\
\text { DBS-6 items } \\
\text { DS-6 items } \\
\text { DBL-8 items }\end{array}$ & CFA \\
\hline [109] & $\begin{array}{l}\text { Initial dimensions of city brand equity } \\
\text { (I stage): perception, preference, satisfaction, attachment } \\
\text { II stage: attachment, image, recommendation, value for money }\end{array}$ & $\begin{array}{c}\text { perception, preference, satisfaction, attachment as elements of CBE } \\
(-) \\
\begin{array}{c}\text { attachment, image, recommendation, value for money as elements } \\
\text { of CBE }(+)\end{array}\end{array}$ & $\begin{array}{l}\text { Initial dimensions: } 47 \text { items } \\
\text { Supported dimensions: } 31 \text { items }\end{array}$ & $\begin{array}{l}\text { regression } \\
\text { analyses }\end{array}$ \\
\hline [110] & $\begin{array}{c}\text { base model: } \\
\text { destination brand awareness (DBA) } \\
\text { destination brand image (DBI) } \\
\text { destination brand quality (DBQ) } \\
\text { destination brand value (DBV) } \\
\text { destination brand loyalty (DBL) } \\
\text { alternative model: DBA, DBV, } \\
\text { destination brand experience (DBEX): DBI, DBQ, }\end{array}$ & $\begin{aligned} & \text { DBA } \rightarrow \text { DBV }(-) \\
& \text { DBI } \rightarrow \text { DBV }(+), \text { DBI } \rightarrow \text { DBL }(+) \text { DBQ } \rightarrow \text { DBV }(+) \\
& \text { DBQ } \rightarrow \text { DBL }(+), \text { DBV } \rightarrow \text { DBL }(+) \text { Alternative model: } \\
& \text { DBA } \rightarrow \text { DBEX }(+), \text { DBEX } \rightarrow \text { DBV }(+) \\
& \text { DBV } \rightarrow \text { DBL }(+), \text { DBEX } \rightarrow \text { DBL (-) }\end{aligned}$ & $\begin{array}{l}\text { 5-point Likert-type scale } \\
\text { DBA-4 items } \\
\text { DBI-4 items } \\
\text { DBQ-4 items } \\
\text { DBV-5 items } \\
\text { DBL-4 items }\end{array}$ & $\begin{array}{l}\text { CFA } \\
\text { SEM }\end{array}$ \\
\hline
\end{tabular}

$(+)$ hypothesis supported, (-) hypothesis not supported. 
Table 3. Findings and practical implications.

\begin{tabular}{|c|c|c|}
\hline Article & Findings/Conclusions Related to CBE & Practical Implications for DMOs, and Destination or City Managers \\
\hline [75] & $\begin{array}{l}\text { Communication in social media, both } \\
\text { controlled and uncontrolled by the } \\
\text { destination organizations, affects the } \\
\text { DBE and its commitment. } \\
\text { Integrated management model } \\
\text { includes communication and BE } \\
\text { management as key factors to } \\
\text { understanding the long-term success } \\
\text { of a destination. }\end{array}$ & $\begin{array}{l}\text { DMOs should } \\
\text { - } \quad \text { define clear strategies for multichannel transmission of traditional, } \\
\text { controlled communication; } \\
\text { - } \quad \text { develop technology-based investments; } \\
\text { - } \quad \text { appoint a community manager, responsible for the effective } \\
\text { communication management to identify the most important } \\
\text { trends, to better understand the customer satisfaction, needs, } \\
\text { expectations of potential tourists, and to track competitive } \\
\text { companies/destinations. }\end{array}$ \\
\hline [76] & $\begin{array}{l}\text { Perception of destination natural } \\
\text { quality has a stronger influence on } \\
\text { destination hedonic value than } \\
\text { destination service quality. } \\
\text { Perception of destination service } \\
\text { quality affect destination functional } \\
\text { value more strongly than destination } \\
\text { natural quality. } \\
\text { Hedonic tourism value had the } \\
\text { greatest impact on brand trust. }\end{array}$ & $\begin{array}{l}\text { DMOs should: } \\
\text { - } \quad \text { use brand awareness tools (traditional and social media) to } \\
\text { promote their destination, } \\
\text { should obtain information about destinations through various } \\
\text { channels and offer high-quality products and services, } \\
\text { - establish quality and service standards, and check them regularly, } \\
\text { - control the pricing policy of companies to check if a product is } \\
\text { sold at the same price to tourists and residents, } \\
\text { - encourage tourists to share their positive experiences and inspire } \\
\text { them to re-visit. }\end{array}$ \\
\hline [77] & 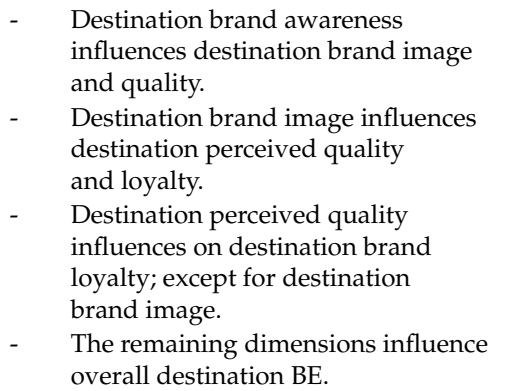 & $\begin{array}{l}\text { - A tourism destination should create an image that is impressive } \\
\text { and unique, complementing the personalities of domestic tourists. } \\
\text { Marketers should carefully consider the beliefs, ideas, styles, } \\
\text { personalities, and needs to satisfy tourists. } \\
\text { Various marketing methods should be used to promote Hoi An, } \\
\text { such as using TV channels, travel magazines, and events. }\end{array}$ \\
\hline [78] & $\begin{array}{l}\text { - The unique selling proposition has an } \\
\text { impact on brand communication and } \\
\text { city branding. } \\
\text { A better unique selling proposition } \\
\text { increases brand communication and } \\
\text { builds a good image of the city } \\
\text { branding as a Craft City. } \\
\text { The unique sales proposition does not } \\
\text { affect BE. Proper communication } \\
\text { is required. } \\
\text { There is a strong relationship between } \\
\text { the city branding and BE }\end{array}$ & $\begin{array}{l}\text { - The city brand communication needs to be improved } \\
\text { - By improving communication, better organization of events, } \\
\text { public relations advertising, direct marketing and word of mouth } \\
\text { marketing, the quality of city branding is improved }\end{array}$ \\
\hline [79] & $\begin{array}{l}\text { Destination brand loyalty has the } \\
\text { highest positive impact on tourism } \\
\text { brand Kurukshetra, followed by } \\
\text { perceived quality and brand image. } \\
\text { Brand awareness has the lowest } \\
\text { positive influence on tourism } \\
\text { brand Kurukshetra. }\end{array}$ & $\begin{array}{l}\text { Kurukshetra city should: } \\
-\quad \text { inform tourists about its marketing campaigns. } \\
\text { - } \quad \text { use better methods such as TV channels, travel magazines, } \\
\text { organizing events, and word-of-mouth. } \\
\text { - improve the product (service) quality with the help of tourism } \\
\text { services-providers such as the government, business firms } \\
\text { and citizens. }\end{array}$ \\
\hline [80] & $\begin{array}{l}\text { Destination brand awareness } \\
\text { influences a destination brand image } \\
\text { and value. } \\
\text { - } \\
\text { Destination brand image influences } \\
\text { destination brand quality, brand value } \\
\text { and loyalty. } \\
\text { Destination brand quality influences } \\
\text { destination brand value and loyalty. } \\
\text { - } \\
\text { Destination brand value influences } \\
\text { destination brand satisfaction } \\
\text { and loyalty. } \\
\text { Destination brand satisfaction } \\
\text { influences destination brand loyalty. }\end{array}$ & $\begin{array}{l}\text { Improvement of destination brand awareness can be achieved by } \\
\text { developing a promotion strategy. } \\
\text { Increasing destination brand image should be done by improving } \\
\text { the image of accommodation services, especially the existence of } \\
\text { host families. } \\
\text { - } \\
\text { Destination brand quality should be improved by increasing the } \\
\text { convenience of using affordable public transport. } \\
\text { Increasing destination brand satisfaction should be achieved by } \\
\text { increasing tourist satisfaction. }\end{array}$ \\
\hline
\end{tabular}


Table 3. Cont.

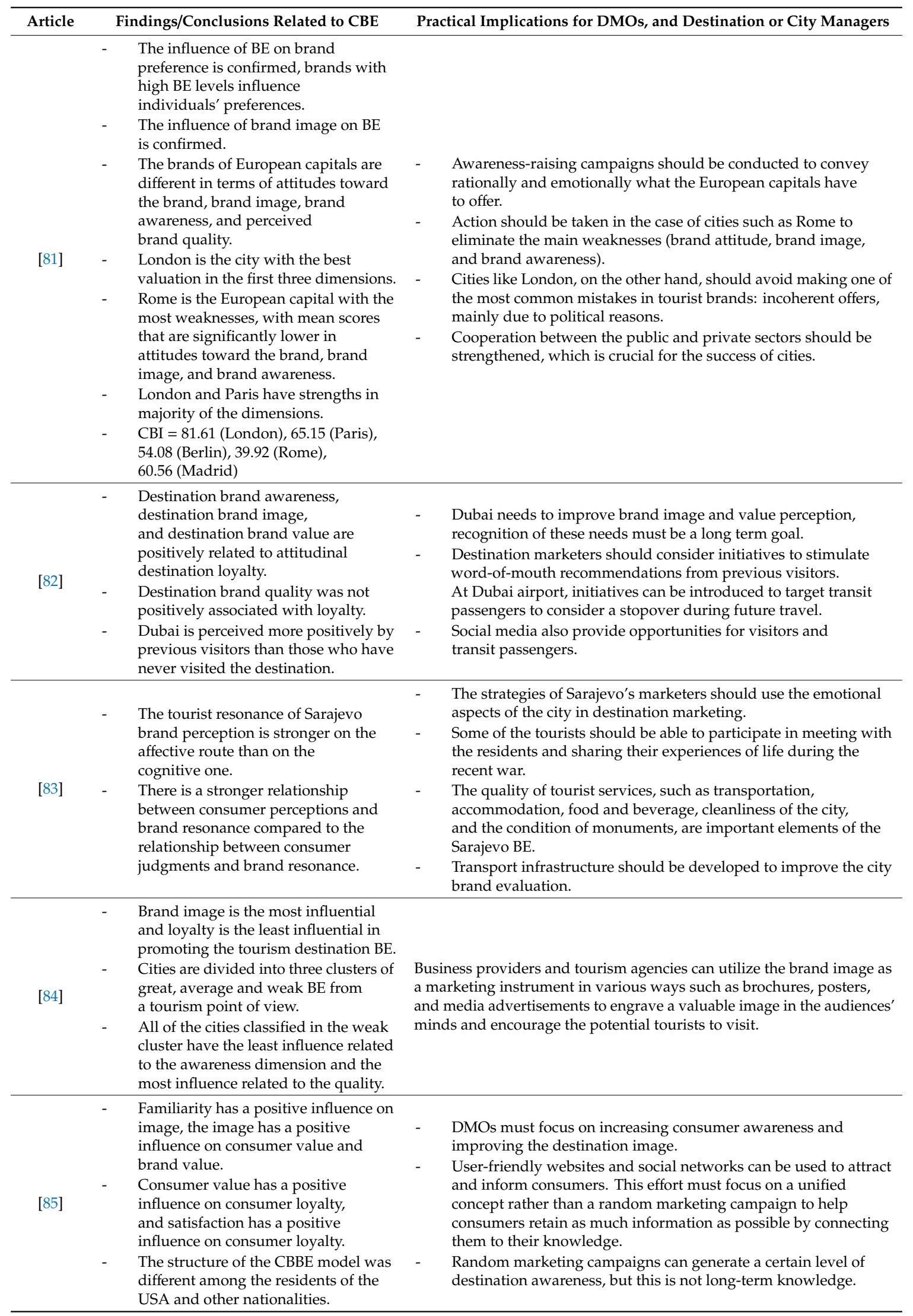


Table 3. Cont.

\begin{tabular}{|c|c|c|}
\hline Article & Findings/Conclusions Related to CBE & Practical Implications for DMOs, and Destination or City Managers \\
\hline [86] & 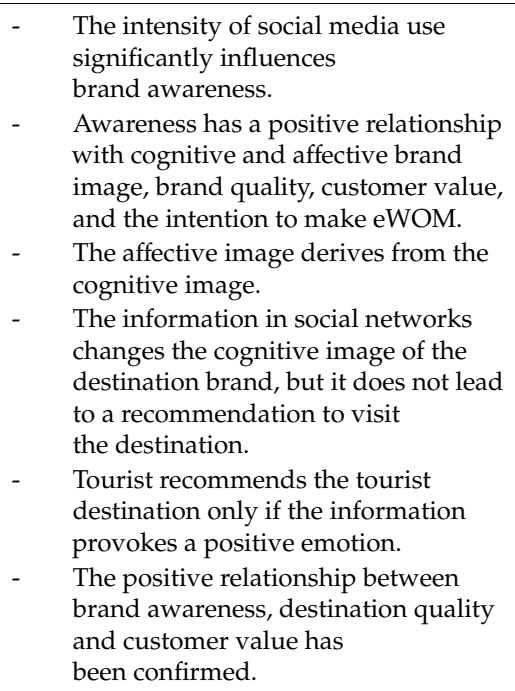 & $\begin{array}{l}\text { Adopting communication on social networks is an opportunity to } \\
\text { generate competitive advantages for the destination and to improve } \\
\text { brand performance. Therefore, destination managers involved in social } \\
\text { media communication } \\
\text { - can improve their positioning against competing destinations. } \\
\text { - } \quad \text { should communicate brand identity through its symbolism, } \\
\text { thus generating a more attractive brand personality. } \\
\text { - must analyze the unique personality of the brand and adopt these } \\
\text { values in online strategy. } \\
\text { encourage tourists to generate more content about the brand by } \\
\text { creating popular hashtags, organizing photo competitions, blogs, } \\
\text { and others. }\end{array}$ \\
\hline [87] & 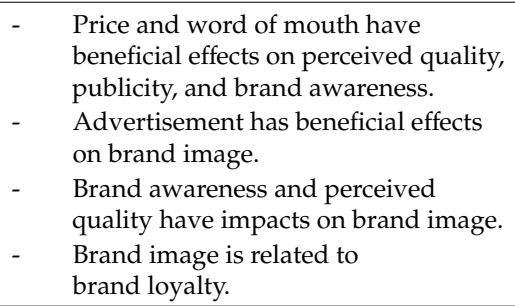 & $\begin{array}{l}\text { Efforts should be made to improve tourism destination perceived } \\
\text { quality and brand image and to cultivate Seoul's brand loyalty as } \\
\text { a tourism destination, } \\
\text { - Interactive communication and word of mouth should increase. }\end{array}$ \\
\hline [90] & 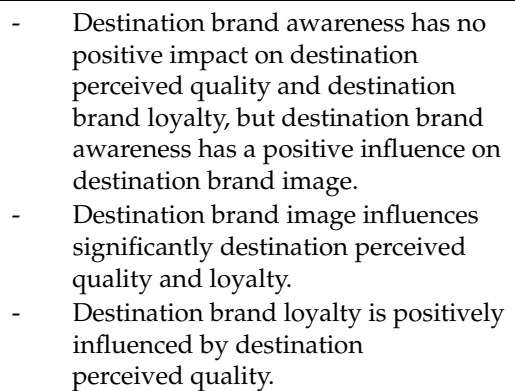 & $\begin{array}{l}\text { City should: } \\
\text { - } \\
\text { conduct marketing campaigns using such methods as TV } \\
\text { channels, tourist magazines, organizing events } \\
\text { carefully learn about the styles, personalities, needs, and adapt } \\
\text { marketing activities to the specifics of tourists. } \\
\text { - } \begin{array}{l}\text { focus its efforts on improving the quality of products (services), } \\
\text { which is most important in brand loyalty. }\end{array}\end{array}$ \\
\hline
\end{tabular}


Table 3. Cont.

\begin{tabular}{|c|c|c|}
\hline Article & Findings/Conclusions Related to CBE & Practical Implications for DMOs, and Destination or City Managers \\
\hline [92] & 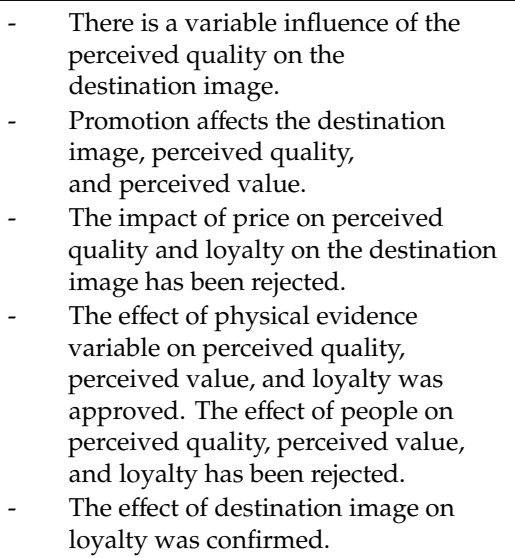 & $\begin{array}{l}\text { - The managers of tourism destinations are advised to continuously } \\
\text { monitor all areas related to tourism. } \\
\text { The owners and managers of residential centers need to be trained } \\
\text { on how to communicate with tourists. }\end{array}$ \\
\hline [93] & $\begin{array}{l}\text { - The city brand identity increases the } \\
\text { strength and effectiveness of urban } \\
\text { branding for the experts' immigration } \\
\text { with higher education. } \\
\text { A city with higher BE has a higher } \\
\text { role to play in city identification and } \\
\text { its residents have a stronger sense } \\
\text { of belonging. }\end{array}$ & $\begin{array}{l}\text { City branding leads to encouraging tourists and experts to visit the city } \\
\text { by building loyalty to the brand and awareness of the city's } \\
\text { development opportunities and attractions. }\end{array}$ \\
\hline [94] & $\begin{array}{l}\text { - Destination brand personality and } \mathrm{BE} \\
\text { have a significant direct effect on the } \\
\text { revisit intention. } \\
\text { - } \\
\text { Destination brand personality has } \\
\text { a positive influence on BE. }\end{array}$ & 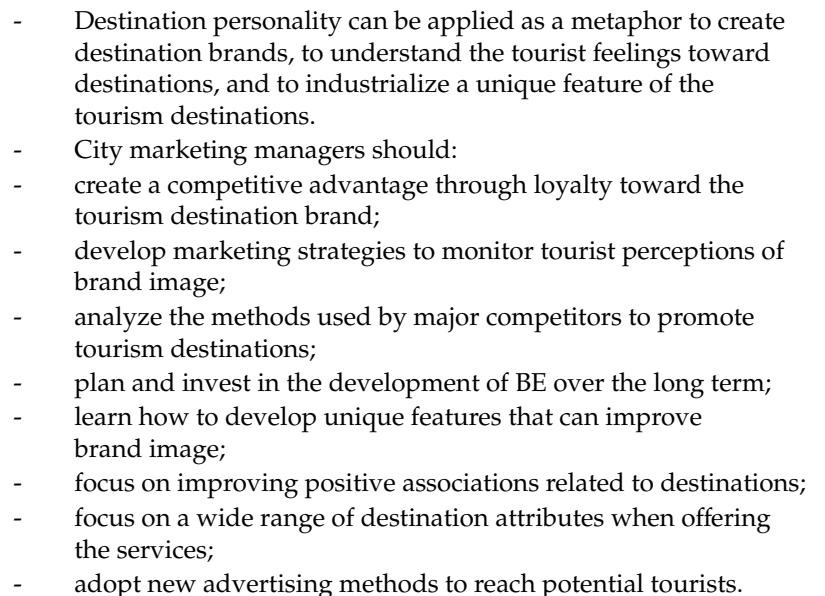 \\
\hline
\end{tabular}


Table 3. Cont.

\begin{tabular}{|c|c|c|}
\hline Article & Findings/Conclusions Related to CBE & Practical Implications for DMOs, and Destination or City Managers \\
\hline [96] & 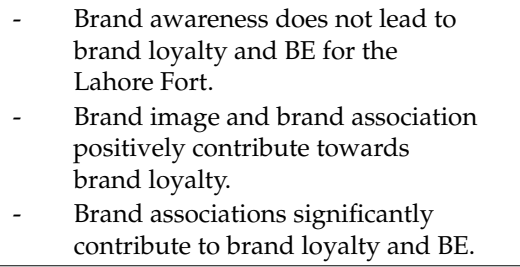 & $\begin{array}{l}\text { - Destination marketers should focus on building a destination } \\
\text { image, a safe environment, local hospitality, comfort for visitors, } \\
\text { and other elements of a brand, such as food items. } \\
\text { - The functional elements, such as parking facilities, cleanliness, } \\
\text { overall guidance provided by staff, and good quality of food } \\
\text { offered can improve the functional imagery of the Lahore Fort as } \\
\text { a destination brand. }\end{array}$ \\
\hline [97] & $\begin{array}{l}\text { - CBBE can lead to increased } \\
\text { destination competitiveness, greater } \\
\text { competitiveness can also result in } \\
\text { stronger CBBE. } \\
\text { - Functional attributes component of } \\
\text { destination competitiveness is the } \\
\text { antecedent of CBBE. }\end{array}$ & $\begin{array}{l}\text { Regional and national tourism authorities should identify the strengths } \\
\text { and weaknesses of the city or destination with regard to its BE } \\
\text { and competitiveness. }\end{array}$ \\
\hline [98] & $\begin{array}{l}\text { - CBE results from the city brand } \\
\text { balance sheet, and the starting point is } \\
\text { investments. When analyzing the } \\
\text { return on investment, the first-order } \\
\text { and second-order effects should be } \\
\text { taken into account. } \\
\text { First-order include number } \\
\text { of website-visitors. } \\
\text { Second-order effects include } \\
\text { intangible assets within the city, e.g., } \\
\text { feeling of ownership, city pride, } \\
\text { storytelling, residential satisfaction; } \\
\text { tangible assets within the city, e.g., } \\
\text { population increase, changes in } \\
\text { income; intangible assets outside the } \\
\text { city, e.g., top-of-mind awareness and } \\
\text { associations toward the city, } \\
\text { and tangible assets outside the city np. } \\
\text { increase in employment }\end{array}$ & $\mathrm{n} / \mathrm{a}$ \\
\hline [99] & $\begin{array}{l}\text { All correlations between the proposed } \\
\text { dimensions were found to be positive and } \\
\text { statistically significant. } \\
\text { Assets have a positive impact on awareness } \\
\text { and quality, while awareness has an impact } \\
\text { on both quality and associations. } \\
\text { Quality and associations have a positive } \\
\text { impact on loyalty. } \\
\text { The significance of cultural brand assets } \\
\text { refers to entertainment/nightlife, festivals, } \\
\text { events, traditions, and street culture. }\end{array}$ & $\begin{array}{l}\text { The DMOs' effort to achieve the desired number of visits in the future } \\
\text { should emphasize the qualitative dimension, adapting it to the context } \\
\text { of its city. } \\
\text { Instead of focusing on investing in high cultural infrastructure, } \\
\text { a cultural brand should be created. } \\
\text { The atmosphere of a cultural destination emphasizes the quality } \\
\text { experiences and indicates the need for an effective organization of } \\
\text { cultural aspects of the destination. } \\
\text { To strengthen loyalty, it is important to remember that international } \\
\text { tourists are more likely to recommend and return to a particular } \\
\text { destination only after reaching quality standards. }\end{array}$ \\
\hline [100] & $\begin{array}{l}\text { There are three categories of place brand } \\
\text { associations: events, city/country name and } \\
\text { famous people } \\
\text { Twitter is not only an interesting way of } \\
\text { understanding place brands, but also to } \\
\text { express and communicate the CBBE. }\end{array}$ & $\begin{array}{l}\text { Place brand managers should: } \\
\text { - approach places and their brands as fragmented phenomena over } \\
\text { which they cannot exercise managerial control in the } \\
\text { traditional sense, } \\
\text { - } \quad \text { underline the need to build a sense of belonging to the brand } \\
\text { community, by maintaining a dialogue with users. }\end{array}$ \\
\hline
\end{tabular}


Table 3. Cont.

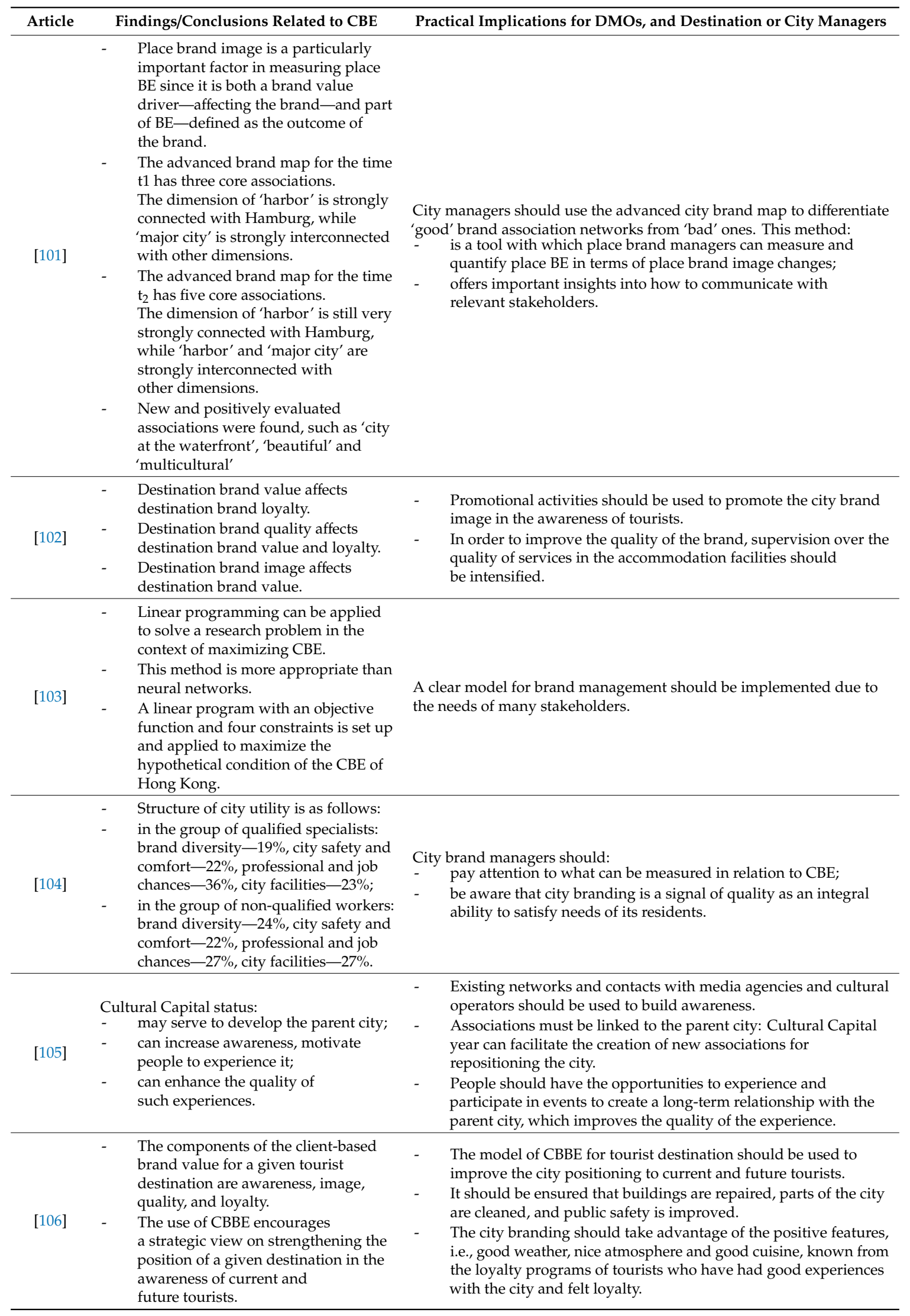


Table 3. Cont.

\begin{tabular}{|c|c|c|}
\hline Article & Findings/Conclusions Related to CBE & Practical Implications for DMOs, and Destination or City Managers \\
\hline [107] & $\begin{array}{l}\text { - The city of Pori and the jazz festival } \\
\text { intertwine in a symbiotic way at every } \\
\text { level of analysis: inside-in, inside- out } \\
\text { and outside-in. } \\
\text { - The image transfer between the event } \\
\text { and the host city is obvious. } \\
\text { - The event contributes to the increase } \\
\text { in destination awareness, perceived } \\
\text { quality, brand associations, } \\
\text { destination loyalty, } \\
\text { and destination positioning. }\end{array}$ & $\mathrm{n} / \mathrm{a}$ \\
\hline [108] & $\begin{array}{l}\text { There are significant, positive relationships } \\
\text { between destination brand awareness, } \\
\text { destination brand image, destination brand } \\
\text { salience, destination brand satisfaction, } \\
\text { and destination brand loyalty. }\end{array}$ & $\begin{array}{l}\text { In city branding, it should be noted that } \\
\text { - } \quad \text { destination brand awareness plays a significant role in influencing } \\
\text { customers to be loyal to the destination; } \\
\text { - } \quad \text { destinations prefer brand image, destination salience, destination } \\
\text { satisfaction as relevant variables related to destination loyalty. }\end{array}$ \\
\hline [109] & $\begin{array}{l}\text { - In the perception of CBE, the strongest } \\
\text { are the elements related to the } \\
\text { perception, preference, satisfaction, } \\
\text { and attachment. } \\
\text { In the perception, image-related items } \\
\text { are the strongest, preference is } \\
\text { replaced by recommendation. } \\
\text { The regression analysis provides } \\
\text { information about the relations } \\
\text { between dimensions that enable the } \\
\text { creation of the ultimate index-CBE. } \\
\text { CBE }=\mathrm{D} 1 \times 83.51 \%+\mathrm{D} 2 \times 9.47 \%+ \\
\text { D3 } \times 4.18 \%+\mathrm{D} 4 \times 2.83 \% \\
\text { Poznań CBE }=0.38, \text { Wroclaw }=0.35\end{array}$ & $\begin{array}{l}\text { - Attachment to a city is central to brand evaluation and BE } \\
\text { followed by recommendation, perception and value for money. } \\
\text { - City managers should identify the weak and strong points of their } \\
\text { CBE and adapt the right tools to the city stakeholders in order to } \\
\text { raise its level. }\end{array}$ \\
\hline [110] & 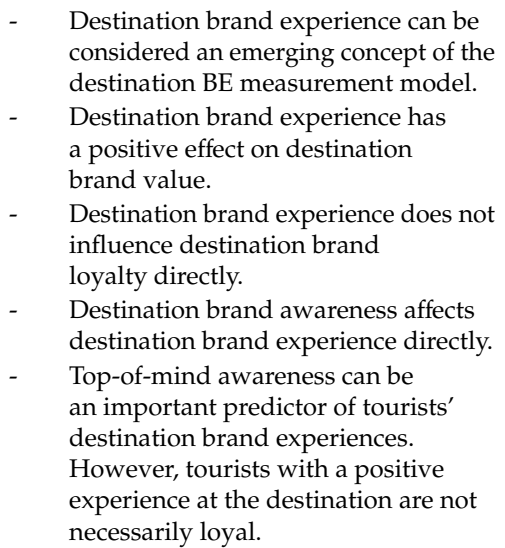 & 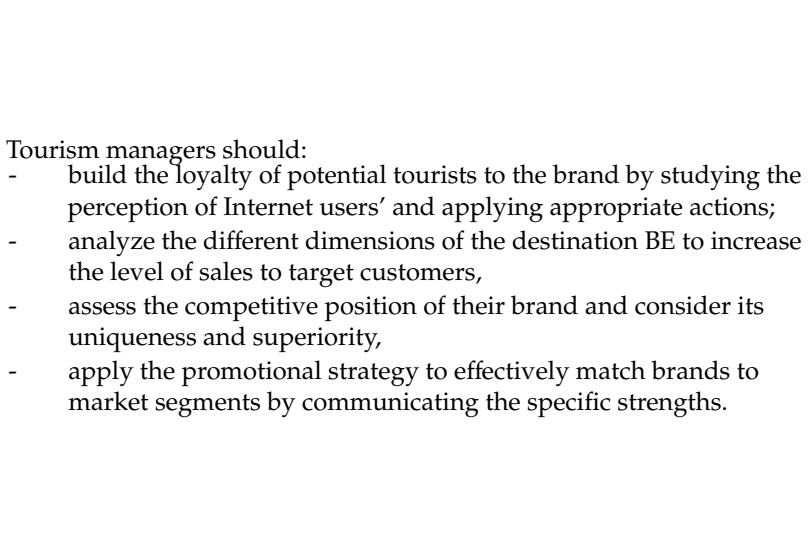 \\
\hline
\end{tabular}

\section{Results}

This Section is divided into three Sub-sections: Section 3.1-general information, Section 3.2-research specifications, and Section 3.3-findings and practical implications.

\subsection{General Information}

Table 1 presents general information related to the author/s, year of publication, research tools, size of sample, sample population and analyzed city or cities.

This SLR includes 36 studies published in 2009-2020 in the following schedule: in 2020-1 study [75], in 2019-five studies [76-80], in 2018 - seven studies [81-87], in 2017-five studies [88-92], in 2016-three studies [93-95], in 2015—two studies [96,97], in 2014—seven studies [98-104], in 2014—four studies [104-107], in 2012—one study [108], and in 2009—one study [109]. 
The questionnaire survey was the most frequently used research method. The research sample had between 150 [79] and 2318 respondents [85]. In the remaining studies, case study [91,98,103,105,107], laboratory experiment [101] and analysis of social media posts [100] were applied.

CBE was analyzed for single cities in 27 studies [75-80,82,83,86,87,89-96,98-102,104-107], two or three cities-in three studies [108-110], and for more cities-in five studies [81,84,85,88,97]. Cities located in Europe [81,83,86,95,97-101,104,105,107,109], Asia [76-80,87,88,90,95,103,106], the Middle East $[76,82,84,89,91-94,102,108]$, and North $[85,110]$ and South America [75] were included in this SLR.

\subsection{Research Specifications}

Table 2 presents research specifications for the studies included in the SLR. The studies were analyzed taking into account the analyzed variables, hypotheses or research questions, applied statistical methods, and research tools. Different numbers of variables were analyzed. For example, in the study on CBE of Alanya, eight variables were included, i.e., destination brand awareness, destination service quality, destination natural quality, destination functional value, destination hedonic value, destination brand trust, destination brand satisfaction, and destination brand loyalty [76]. In the study of the brand of Khalkhal, 10 variables were considered, i.e., perceived value, destination brand loyalty, destination $\mathrm{BE}$, destination perceived quality destination, destination brand image, price, distribution, sale promotion, physical evidence, and people.

In 21 cases, confirmatory factor analysis (CFA) and SEM (Structural equation modeling) were used for statistical analysis for the cities studied individually and comparisons were made. The statements were evaluated on a 5, 7 or 11-point Likert scale. An interesting solution was applied for five European capitals (London, Paris, Berlin, Rome, and Madrid), for which the City Brand Index (CBI) was calculated, allowing BE comparisons of the analyzed cities. Four variables were used to calculate the CBI, i.e., attitude toward the brand, brand image; brand awareness; and perceived quality (each of them rated on a scale from 0 to 10) [81]. In turn, the study of 16 Iranian cities took into account four factors, i.e., brand image, brand awareness, perceived quality, and brand loyalty; additionally, cluster analysis was conducted. Cities were divided into three clusters of great, average, and weak BE of the tourism destinations point of view. Different dimensions of the BE of tourism destinations were compared with each other within three clusters through discriminant analysis [84].

\subsection{General Finding and Practical Implications}

Table 3 presents findings and conclusions related to CBE as well as practical implications for destination marketing organizations (DMOs), destination or city managers, and others. The findings/conclusions refer to the determinants of $\mathrm{CBE}$, that is the research methods used. They also indicate the importance of CBE in branding cities. Practical recommendations are included almost in all articles included in the SLR. Only two studies did not include practical recommendations.

\section{Discussion}

\subsection{CBE as a Research Domain}

In this study, theSLR was conducted for 36 studies related to CBE or DBE, focusing exclusively on cities. In the studies included in the SLR, single cities were analyzed in 27 studies [75-80,82,83,86,87,89-96,98-102,104-107], and two or three cities were compared in three studies [108-110]. In the remaining studies, CBE was analyzed for a larger number of cities $[81,84,85,88,97]$. The analysis was carried out for the European $[81,83,99]$ and Asian $[87,103]$ capitals, as well as for the largest and most populated cities $[82,85,87,103,110]$, and those known for their unique tourist attractions $[81,82,86,87,97,99,103]$. In most of the studies included in the SLR, a questionnaire survey was used as a research method (29 studies). Case studies [91,98,103,105,107], a laboratory experiment [101] and social media post analysis [100] were also applied.

The application of BE for the city brands is based on two reasons. One of them is to use this concept for tourist destinations. In this aspect, the following terms are used: destination BE [111,112], 
customer/consumer-based BE [111,113-117], tourism destination BE [118-120], place BE [121,122] for Australia [18,111], Pakistan [114], Chile, Brazil and Argentina [115], Puerto Rico and US Virgin Islands [117], Slovenia [116,118,123], Switzerland and Austria [124], and Korea [120]. Other destinations, i.e., Kashmir valley [125], wine tourism destinations in Spain [119], mountain tourism destinations in the Patagonia region of Argentina [113], and Southern U.S. states [121] were also considered. In the above-mentioned studies, the following elements of BE were analyzed: brand/destination awareness [114,116,118-121,123-125], brand/destination image [114,116,118,119,123-125], brand/destination loyalty [114,116,118,119,121,123-125], brand associations [121,124], brand/destination perceived quality [116,121,125] or brand/destination quality $[114,118,123]$, perceived value [119], or value [120], as well as social media [114]. Special elements were also considered, including nature, culture, infrastructure, socioeconomic environment, atmosphere, and social conditions [119] as well as preference, uniqueness, and price [120].

The second reason for applying the BE concept to cities is to transfer theories from corporate brands to city brands. In the literature, the similarities were grouped into categories indicating an integrated approach dedicated to managing city brands. The following groups were distinguished: vision and strategy, internal culture, local communities, synergies, infrastructure, cityscape and gateways, opportunities, and communications [19]. When making such a transfer, the specifics of the place/destination/city should be taken into consideration. This is because various indications refer to geographical nomenclature, product-place co-branding, and place branding [20]. At the same time, the use of systems and structures of generic branding models increases the effectiveness of activities undertaken within the city branding. Such actions may consist, for example, in distinguishing visible elements (hotels, airports and trains culture, arts, history nightlife, shopping, safety, cleanliness, transportation, education, health, housing, employment, business, infrastructure) from the invisible related to (1) image, style and personality, (2) awareness, recall, recognition, (3) perceived quality, consideration, (4) emotional and self-expressive elements, as well as (5) loyalty, and engagement [21]. Another statement defines tangible assets as perceived quality, impression, and promotion, with intangible elements relating to awareness, heritage, personality, reputation, and trustworthiness [126].

In the scientific literature, there are various measures related to city branding, these are the City Brand Hexagon and City Brand Index [127], CBE Triangle [128], Tourist-based Brand Equity Pyramid [129], European City Brand Barometer [130] or relating to the sustainability of the city called Green Brand Hexagon [131,132]. The City Brand Hexagon consists of six elements: a presence related to the international status and position of the city, a place that focuses on the perception of physical aspects, the potential for economic and educational opportunities, the pulse associated with the urban lifestyle, inhabitants in the context of friendliness, and prerequisites related to the perception of the basic characteristics of the city [127]. An interesting concept is also the CBE triangle of such three elements: city brand elements (events and activities, history and heritage, graphics and symbols, process and institution, as well as artifacts and spatial planning), city brand measurement (quantitative, qualitative and mixed), and the city brand impact (economic, socio-political, image-identity) [128]. One of the most holistic approaches refers to CBBE for tourist destinations including four elements: destination brand awareness, perceived quality, destination brand image, and destination loyalty (cognitive, affective, conative and action). The destination choice flowchart consists of the following sequences: market (all existing destinations), consideration (awareness and unawareness set), evaluation (evoked, surrogate, and exclusion set), constraints (dream, available and unavailable set), choice and post visit-evaluation. This approach focuses on the two most important destination brand elements: favorite and dream destination [23].

\subsection{Major Waves of CBE Research}

The studies included in the SLR are characterized by different research topics resulting from different brand equity approaches analyzed in relation to the city. Five waves of researchers' interest can be distinguished (Table 4). The first two waves refer to different ways of perceiving city brand loyalty. In the first wave, loyalty is seen as a result of CBE and, at the same time, as a result of all 
factors determining CBE. The second wave refers to considering loyalty as one of the CBE factors, alongside the brand image, brand awareness, and perceived brand quality. The third wave is related to communication with tourists and the importance of communication process in shaping CBE. This group examined social media and advertising, pointing to the need of selecting appropriate communication tools to show all the attributes of the city, including elements that make it unique and original. The fourth wave refers to unique city brand assets that are tourist attractions, and refer to history, culture, special events, etc. Unique city brand assets influence CBE directly or indirectly, shaping the image of the city, and brand quality. The last wave indicates a willingness to quantify CBE in order to make city comparisons. At the same time, the quantitative expression of the city brand equity may, in the opinion of city managers, be an element facilitating the management of the city brand.

Table 4. Major waves of city brand equity (CBE) research.

\begin{tabular}{|c|c|c|c|}
\hline & Waves of CBE Research & Main Topics Analyzed & Studies Included in SLR \\
\hline 1 & $\begin{array}{l}\text { Loyalty as a result of } \\
\text { factors determining CBE } \\
\text { directly or indirectly }\end{array}$ & $\begin{array}{c}\text { Brand loyalty is the result of: } \\
\text { brand satisfaction } \\
\text { brand trust } \\
\text { perceived brand value } \\
\text { perceived brand quality } \\
\text { brand awareness } \\
\text { brand image } \\
\text { brand identity } \\
\text { consumer value } \\
\text { brand personality } \\
\text { brand associations } \\
\text { brand salience } \\
\text { brand experience }\end{array}$ & $\begin{array}{c}{[76,80,108]} \\
{[76]} \\
{[80,82,85,92,101,110]} \\
{[80,82,85,87,88,92,99,101]} \\
{[82,87,88,99,108,110]} \\
{[82,85,87,88,92,108]} \\
{[88]} \\
{[83]} \\
{[85]} \\
{[93]} \\
{[99]} \\
{[108]} \\
{[110]}\end{array}$ \\
\hline \multirow{3}{*}{2} & \multirow{3}{*}{$\begin{array}{l}\text { Loyalty as one of the } \\
\text { determinants of CBE }\end{array}$} & $\begin{array}{c}\text { Brand loyalty, perceived quality, brand awareness and } \\
\text { brand image influence CBE. }\end{array}$ & {$[77,79,81,84,90]$} \\
\hline & & $\begin{array}{l}\text { Brand loyalty, brand awareness, brand image and brand } \\
\text { associations influence CBE. }\end{array}$ & [96] \\
\hline & & $\begin{array}{l}\text { Brand loyalty, brand awareness, brand image, and quality } \\
\text { influence CBE. }\end{array}$ & [106] \\
\hline \multirow{4}{*}{3} & \multirow{4}{*}{$\begin{array}{l}\text { Communication and its } \\
\text { role in shaping CBE }\end{array}$} & $\begin{array}{l}\text { DMO- and tourist-generated social media communication } \\
\text { influence destination awareness and image directly, } \\
\text { and destination quality indirectly. }\end{array}$ & [75] \\
\hline & & Proper communication influences city branding and CBE. & [78] \\
\hline & & $\begin{array}{c}\text { Advertising influences brand image and promotion } \\
\text { brand awareness. }\end{array}$ & [87] \\
\hline & & Social media influence CBE. & {$[75,86,100]$} \\
\hline \multirow{6}{*}{4} & \multirow{6}{*}{$\begin{array}{l}\text { Unique city assets as } \\
\text { drivers of CBE }\end{array}$} & $\begin{array}{l}\text { Unique city brand assets affect the city image directly and } \\
\text { perceived quality indirectly. }\end{array}$ & [77] \\
\hline & & Unique city brand assets influence BE. & {$[91,98]$} \\
\hline & & Unique city selling offers influence city branding and CBE. & [78] \\
\hline & & Historical determinants are important elements of CBE. & {$[83,94,95,99]$} \\
\hline & & $\begin{array}{l}\text { Cultural brand assets are one of the elements } \\
\text { influencing CBE. }\end{array}$ & {$[89,93,95,99,105,107]$} \\
\hline & & Functional city attributes influence $C B E$. & {$[97,104]$} \\
\hline \multirow[t]{2}{*}{5} & \multirow{2}{*}{ CBE indicators } & $\begin{array}{l}\text { City Brand Index is calculated using the BE dimensions: } \\
\text { attitude toward the brand, brand image, brand awareness } \\
\text { and perceived quality. }\end{array}$ & {$[81]$} \\
\hline & & $\begin{array}{l}\text { CBE index is calculated using BE dimensions: attachment, } \\
\text { image, recommendation and value for money. }\end{array}$ & [108] \\
\hline
\end{tabular}




\subsection{Factors Determining $C B E$}

The identification of CBE determinants is the answer to the first research question. The main factors include brand image, brand quality, and brand awareness (Table 5). They were analyzed in 24, 21 and 19 studies, respectively. Brand loyalty was also frequently analyzed; however, in some cases brand loyalty was treated-according to Aaker's model [25]—as an element of BE, similarly as perceived quality, brand association and brand awareness. In other cases, brand loyalty was analyzed as a result of actions taken and the result of specific strategic activities applied in the area of city branding. This is consistent with the understanding of BE in the Keller's model [26].

Table 5. Factors determining CBE analyzed in studies included in the SLR.

\begin{tabular}{|c|c|}
\hline Factors Determining CBE & Number of Studies Analyzing This Factor \\
\hline brand image & 24 \\
\hline brand quality & 21 \\
\hline brand awareness & 19 \\
\hline brand loyalty (result of BE and other activities) & 14 \\
\hline brand loyalty (factor) & 7 \\
\hline brand satisfaction & 5 \\
\hline brand attributes & 4 \\
\hline brand value & 3 \\
\hline brand attachment & 3 \\
\hline brand identity & 3 \\
\hline brand resonance & 3 \\
\hline brand assets & 3 \\
\hline communication & 3 \\
\hline consumer value: perception of value for money & 3 \\
\hline brand judgment & 2 \\
\hline brand performance & 2 \\
\hline brand familiarity & 2 \\
\hline brand experience & 2 \\
\hline brand personality & 2 \\
\hline price & 2 \\
\hline brand trust, brand feeling & 1 \\
\hline brand salience & 1 \\
\hline brand engagement with destinations & 1 \\
\hline tourist-generated social media communication & 1 \\
\hline advertisement, promotion, sale promotion & 1 \\
\hline marketing and administrative expenditure & 1 \\
\hline physical evidence, people & 1 \\
\hline distribution, and unique selling proposition & 1 \\
\hline customer feelings about the vacation experience & 1 \\
\hline positioning, favorability, uniqueness & 1 \\
\hline city diversity, city facilities, tradition & 1 \\
\hline city safety and comfort & 1 \\
\hline
\end{tabular}

Due to the fact that three factors (brand perceived quality, brand image and brand awareness) were most often analyzed as determining elements of $\mathrm{CBE}$, a detailed description of them is provided below. Brand assets described both as separate factors or appearing as elements influencing the brand image and brand associations are discussed separately. At the same time, attention was paid to the sustainability elements concerning the specific determinants of $\mathrm{CBE}$, which were included in the second and third research questions.

\subsubsection{City Brand Image}

In this SLR, the city brand image appeared in 24 studies as destination brand image [77,80,82,90,92,102,108,110], brand image [79,81,84,86-88,95,96,100], and destination image [75,83]. It was analyzed in different ways by 
quantitative evaluation of statements and qualitative analysis of dimensions and factors determining perception of the city and its brand.

In the scientific literature, the brand image is defined as a separate set of associations related to the brand, held in the memory of the consumer [133], while the destination image is perceived as an interactive system of thoughts, opinions, feelings, visualizations and intentions concerning tourist destinations [134]. It is created by historic charms, the number of cultural and festival events, good museums and art galleries, outstanding scenery, good state parks and forests, and great places of outdoor activities [133]. Destination brand image perceived as perception of a place in the tourist memory [135], combines affective and cognitive structures [136]. Destination imagery refers also to the work and long memory with attention to the perception of destination imagery analyzed via functional, psychological, common and unique characteristics as well as in a holistic approach [23].

Studies included in this SLR indicated interesting cultural and historical attractions [82,102], an interesting brand [81], advantage, and originality over other destinations and cities [75]. Comparisons of tourist's personality and city brand $[75,77,82,90,102]$ as well as the image of the tourist and the city image $[77,81,82,90]$ were also referred to. The literature emphasizes that brand image includes the features and advantages that make a brand unique [137]. This is due to the fact that brand image is associated with personality [138]. Additionally, brand personality enables brand creation taking into account emotional links [2]. However, the brand image, apart from the personality, also includes the value association, and associations with the organization [139].

Some of the studies in this SLR have included the assessment of the city brand divided into affective image and cognitive image. In the case of Sarajevo city, the following statements were assessed as affective image: enjoyable, exciting and tourist-friendly city, as well as friendly and ready to help residents. In the group of creatures concerning the cognitive image, a combination of old and new buildings, a clean and uncontaminated city and the nature of the city were assessed [83].

The evaluation of the city brand image was often related to qualitative aspects. In the case of the city of Khalkhal, constructs such as: the weather of the city, the availability of travel information, the adventure atmosphere of the city, the hospitality rate of the residents and the natural attractions and beautiful landscapes of the city, were taken into consideration [92]. In some cases, brand image has included brand attitude and brand quality incorporating such elements as historical and aesthetical appealing, cleanliness, accommodation, infrastructure, shopping facilities, halal food, and culture [96].

In the evaluation of the image of Valencia, several levels were analyzed, referring to (1) cultural image, including cultural and historic city, interesting museums, tourist attractions; (2) nature, including parks, nature zones, environment of Valencia (villages and nature), and attractive beaches; (3) attractions and leisure, with shopping facilities (shops, shopping centers), events (fairs, festivals, etc.), and local gastronomy, and (4) Mediterranean city, considered in terms of tourists' perception of the city [86]. Similar aspects were taken into account when analyzing the image of Bandung city in Indonesia. In this case, offerings focusing on the historical aspects (museum and cultural/historical events) were associated with city ambiance, reflecting such features as atmosphere, weather, shopping facilities, entertainment and nightlife [106].

In the case of the city of Perm, the city image was evaluated on three levels. The low level indicated a negative image, and the city was characterized as dull, grey, and boring. The medium level was explained in the context of neutral image of the city, indicating the terms 'nothing to boast for, nothing to complaint for'. The high level, however, indicated a very attractive image of the city [104].

The above-mentioned factors influencing brand image are reflected in the literature [140-145]. One of the most extensive lists presents dimensions and attributes determining the perceived tourist destination image. This list includes the following elements: natural resources (e.g., weather, richness of the scenery, variety and uniqueness of flora and fauna), general infrastructure (e.g., health services, public and private transport, quality of roads, airports, commercial infrastructure), tourist infrastructure (e.g., hotel and self-catering accommodation, restaurants, tourist centers), political and economic factors (e.g., political stability and tendencies, economic development, prices, safety), culture, history 
and art (e.g., museums, historical buildings, monuments, religion, folklore, festivals, concerts, etc.), tourist leisure and recreation (e.g., theme parks, entertainment and sport activities), natural environment (beauty of the scenery, cleanliness, overcrowding, air and noise pollution), social environment (e.g., hospitality and friendliness of the local residents, language barriers, quality of life), and atmosphere of the place (e.g., destination described as luxurious, fashionable, exotic, relaxing, stressful, attractive, interesting, enjoyable, pleasant, etc.) [146].

\subsubsection{City Brand Quality}

Quality as an element of BE in relation to the city was analyzed in 22 studies as destination perceived quality $[75,77,90,92]$, perceived brand quality $[79,87,93]$, destination brand quality $[80,82,99,102,110]$, perceived quality $[81,84,88,89,107]$, and brand quality $[86,96]$. In one case, a division was made between destination service quality and destination natural quality [76]. In the literature, brand quality such as perceived quality is defined as the judgment made by the consumer regarding the overall perfection or superiority of a product. [147]. It may refer to such dimensions as: performance, reliability, durability, features, conformation quality, serviceability, as well as style and design [148]. For tourist destination, brand quality is defined as a strong and influential component of CBBE [123] and travelers' perception of a destination's ability to fulfill their expectations [149]. In this respect, it may refer to hotel accommodations, shopping entertainment [149] or in general do destination facilities [111].

Environmental aspects were important elements of the city brand quality assessment, in some cases influencing the brand image of the city. For example, in relation to Bandung city, environmental pollution, level of cleanliness and quality of infrastructure were assessed by tourists as unsatisfactory. This indicates that the level of quality as BE element depends on city pollution, infrastructure quality and cleanliness. Tourists visiting Bandung city felt unsatisfied with the polluted environment as the city is getting more crowded, and full of vehicles. They also pointed a lack of proper maintenance for public buildings and issues relating to public safety [106]. Similarly, in the case of Dubai, the quality aspects related to levels of cleanliness and quality infrastructure [82].

In the study on the city of Perm, the city's quality attributes were associated with ecology ratings; a low level referred to a highly polluted city, while a high level meant low pollution and an ecologically clear city. The development of transport was also analyzed, indicating that a low level of transport infrastructure meant uncomfortable road junctions, uncomfortable traffic net, limited railways and traffic. The development of the high level of infrastructure indicated the possibility of easy access to this city by various means of communication while at the same time providing good internal communication solutions [104]. Similar aspects were analyzed for Iranian cities, indicating clean air, high-quality transportation infrastructures, road infrastructure quality, and hygienic environment. Attention was also drawn to persistence (sustainable) quality [84].

In assessing the quality of cities, an important element was the availability of parks and green zones. In case of Perm city, the presence of parks was analyzed at low and high levels. A low level meant no green zones inside the city, while forests and rivers were hardly available. A high level indicated a large number of parks and available green zones [104]. As indicated by the studies quoted in the literature, the presence of natural green areas and parks is important in relation to destination $\mathrm{BE}$ as an important element of the dimension defined as "nature" [119]. Urban nature is important from an environmental and ecological point of view; it also provides psychological and emotional benefits to residents and tourists [150]. Environmental, economic, social and cultural benefits of urban nature influence four elements of city branding, including global competitiveness, social cohesion, lifestyle changes, and climate change [151]. At the same time, a "green" or environmentally sustainable city brand contributes to sustainable development [152]; additionally, a green city brand determines the willingness to stay in a city [153].

In some cases, sustainable elements were present in the evaluation of the quality of experience, which is the brand of performance. For the city brand of Sarajevo, transportation infrastructure, hotels and restaurants in terms of customer service and facilities, the variety of food and beverage 
choices, tourism information services, opportunities for entertainment and sport activities, as well as communication problems with locals, were considered [83]. The experience as an element of quality was also analyzed in the case of two American cities: Las Vegas and Atlantic City [110].

Quality aspects were also taken into account in the case of Lahore in such BE element as brand association, indicating high-quality services, high-quality tourist attraction and the overall picture of high quality [96]. The aspect of high-quality tourist attractions was also analyzed in the case of Shiraz [102] and Danang [90]. The quality of the experience is also determined by authentic cultural experience, which is particularly evident with Rome brand quality. The impact on brand loyalty considered as a willingness to recommend and visit again is achieved [99].

The quality of Valencia brand was analyzed quite differently. It indicated a very good tourist offer, a range of lodgings, good quality of local infrastructure and transport with a sense of security and satisfaction [86]. In the case of Las Vegas and Atlantic City, it has been pointed out that cities/destinations should provide tourism offerings of consistent quality and tourists can expect superior performance. As a result, destination performs better than other similar destinations [110]. The aspect of comparing performance with other destinations and the expectations of tourists for the best performance of the destination was also analyzed in the case of Shiraz [102] and Danang [90].

The quality of the Dubai brand in terms of the offer for tourists was analyzed as a high level of personal safety and high-quality of accommodation [82]. The quality assessment of the Seoul brand analyzed whether the quality of tourism products (accommodation, transportation, shopping, etc.) are outstanding. Attention was also paid to whether Seoul continuously provided high-quality tourism products and services [87]. Similarly, high-quality services and the high quality of accommodation centers were considered for 16 Iranian cities [84].

An interesting method of analyzing city brand quality was used for Alanya in Turkey. A differentiation was made for destination service quality, including accommodation, transportation, cleanness, hospitality activities, amenities, language and security and destination natural quality with climate, historical and cultural attractions. Studies have shown that the natural quality perceptions are mostly related to the emotion-based situations for individuals such as the atmosphere (climate, historical attractions) and the culture, they could have a relatively stronger impact on destination hedonic value perceptions [76].

\subsubsection{City Brand Awareness}

City brand awareness or destination brand awareness related to cities was analyzed in 19 studies, including destination awareness [75,107], destination brand awareness [76,77,80,82,90,102,108,110] and brand awareness $[79,81,84,86-89,93,96,99]$.

In the scientific literature, brand awareness is described as familiarity, a signal of substance/commitment or an 'anchor to which other associations can be attached' [25]. It is also perceived as a necessary element for the image formation [26] representing the strength of the brand's presence in the mind of the target audience [139]. In the case of tourist destination, brand awareness is defined as the image of a destination existing in the minds of potential travelers [154]. Brand awareness of tourist destinations is also defined as the ability of tourists to recall a specific tourism destination among various locations, and to distinguish it from others $[155,156]$. Destination marketing aims to raise awareness of a destination by creating a unique brand [157].

In studies included in this SLR, city brand awareness was analyzed by evaluating statements regarding the perception of the city as famous, unique $[77,81,82,86,89,90,99,110]$, and tourist destination [75], with good name and reputation $[77,82,86,89,90,98,110]$, recall from memorizing the name of the city $[77,87,89,90,99]$, or its character $[77,82,86,89,90,99,110]$, being aware of the existence of a given city and its attributes $[75,84,87]$, being able to compare and distinguish this city from another $[84,87]$, logo or symbol of the tourism destination [84], knowledge of the city's advertisements [82], and ease of recognition [81].

In discussing the above results, it should be noted that brand awareness begins to appear after gaining more knowledge about the brand at the beginning, then the learning process begins; thereafter, 
the attractiveness of the brand begins to appear. These elements will create connotations, shape attitudes, and, as a result, positive attitudes can bring BE [158]. This aspect relates to the understanding of brand awareness as the ability to remember a brand logo [159]. At the same time, it should be noted that CBDBE creates and maintains brand awareness, and positive feelings, which could increase the possibility of revisiting [75].

\subsubsection{Specific City Brand Assets}

Brand assets are important elements for creating CBE. In the case of Rome, brand assets have been analyzed in order to assess the city as unique. Attention was paid to entertainment/nightlife options, cultural festivals, traditions, cultural events, street culture, monuments/heritage sites, cuisine, art centers, museums and contribution to world heritage. The greatest importance of cultural brand assets was related to entertainment/nightlife, festivals, events, traditions and street culture realized in a city known for its long history. These assets make Rome a famous cultural destination that immediately comes to mind when asked about culture and create city brand awareness [99]. Literature points to the importance of cultural assets indicating the existence of an urban cultural appeal matrix including hard and soft cultural assets as well as local vs. global nature of the key cultural variables [160].

An example of cyclical cultural events affecting the development of the city is the Pori Jazz Festival organized since 1966 in Pori in Finland. The development of the city and the festival took place simultaneously. Moreover, the transfer of image between the festival and the host city has been observed. The city has developed on a political, economic, environmental and social level, and the jazz festival has gained international recognition [107]. The literature shows the importance of music or film festivals and artistic performances in the promotion of cities. In this regard, festivals/artistic performances can be used by city tourism managers to promote city as a corporate brand [7,161-164]. However, this depends on several factors, including the size of the festival, its importance, uniqueness, and frequency [161] as well as cooperation with tourist agencies and the government [165]. Local or minor theme festivals cannot be used as marketing tools to promote the city [161].

An important way to strengthen the CBE is to include the city on the UNESCO World Heritage List. This list includes 1121 properties of historical (869 properties), natural (213) and mixed (39) importance [166]. According to the mission of UNESCO and World Heritage Convention, "heritage is our legacy from the past, what we live today, and what we pass on to future generations" [167]. Among the studies in the SLR, the positive impact of brand awareness on brand image and perceived quality, brand image on perceived quality as well as brand loyalty and perceived quality on brand loyalty was found in the case of Hoi An City (Vietnam) included in the UNESCO list [77].

In turn, cultural differences in Istanbul, another UNESCO-listed city, have a significant impact on tourists' satisfaction and their desire to visit the city again. Cultural assets, such as monuments, heritage attractions, museums, art centers, cultural events and festivals, point to a competitive advantage. At the same time, associations with brands help build favorable attitudes and beliefs towards their brands to ensure a higher BE [89]. Similar brand assets were analyzed in the case of Lahore Fort on the UNESCO list [96]. However, in the case of Lulea, which is on the UNESCO list due to its historical old town, environmental considerations are also important. The city of Luleå comprises 1300 islands, and the mix of salt and fresh water makes the archipelago very special and unique [95].

The inclusion of the city in the UNESCO list increases its attractiveness and contributes to the development of cultural tourism. In this aspect, the intention of UNESCO is to develop sustainable tourism [168]. It is also fundamental to preserve its historical and artistic inheritance and, at the same time, to encourage visits to that area [169].

Another example of the city asset creating BE is the awarding of a prestigious title or the location of prestigious events in it. Among the cities surveyed, Turku in Finland, which has become European Capital of Culture, is an example. It is a prestigious title that allows the development of CBE through multifaceted activities, also in social, cultural and economic areas. Cities applying for this title must meet requirements, including a high-quality program of events; the commitment of public authorities, 
especially in terms of financing, and the involvement of social and economic stakeholders [105]. Similar experiences are quoted in literature for other cities [170].

\section{Conclusions}

Based on the SLR, it should be noted that there are many factors influencing CBE. The main factors include brand image, brand quality, and brand awareness. Each of these factors refers to a number of elements influencing the perception of the city's brand. They concern cultural and environmental aspects and are also related to services provided to tourists. Environmental factors related to air pollution, infrastructure quality, presence of green areas, quality of urban transport, cleanliness and safety are important in perceiving the quality of the city brand. The brand assets also determine CBE and relate to quantitative and qualitative elements confirming the uniqueness of the city and its brand.

The SLR fills a gap in terms of publications on CBE. However, it has limitations on the exclusion criteria used, which does not consider conference materials, books, dissertations and others. However, this is due to the rules applicable to all SLRs.

This type of research on CBE should be continued, trying to introduce quantitative indicators to compare the BE of different cities over a given period of time, as well as to analyze changes over time. This may prove to be particularly important to ensure sustainable city development.

Supplementary Materials: The following are available online at http://www.mdpi.com/2071-1050/12/19/7858/s1, Table S1: List of studies included in the systematic review (in alphabetic order).

Funding: The Article Processing Charge was financed by the Polish Ministry of Science and Higher Education within funds of Institute of Human Nutrition, Warsaw University of Life Sciences (WULS) for scientific research.

Conflicts of Interest: The author declares no conflict of interest.

\section{References}

1. Simona Moise, M.; Gil-Saura, I.; Seric, M.; Ruiz Molina, M.E. Influence of environmental practices on brand equity, satisfaction and word of mouth. J. Brand Manag. 2019, 26, 646-657. [CrossRef]

2. Biel, A.L. How brand image drives brand equity. J. Advert. Res. 1993, 32, 6-12.

3. Seric, M.; Gil-Saura, I.; Molla-Descals, A. Can advanced technology affect customer-based brand equity in service firms? An empirical study in upscale hotels. J. Serv. Theory Pract. 2016, 26, 2-27. [CrossRef]

4. Hyun, S.S.; Kim, W. Dimensions of brand equity in the chain restaurant industry. Cornell Hosp. Q. 2011, 52, 429-437. [CrossRef]

5. Tong, X.; Hawley, J.M. Measuring customer-based brand equity: Empirical evidence from the sportswear market in China. J. Prod. Brand Manag. 2009, 18, 262-271. [CrossRef]

6. Vallaster, C.; Wallpach, S.; von Zenker, S. The interplay between urban policies and grassroots city brand co-creation and co-destruction during the refugee crisis: Insights from the city brand Munich (Germany). Cities 2018, 80, 53-60. [CrossRef]

7. Ooi, C.S.; Pedersen, J.S. City branding and film festivals: Re-evaluating stakeholder's relations. Place Brand. Public Dipl. 2010, 6, 316-332. [CrossRef]

8. Riza, M.; Doratli, N.; Fasli, M. City branding and identity. Procedia Soc. Behav. Sci. 2012, 35, $293-300$. [CrossRef]

9. Kavaratzis, M. City Marketing: The past, the present and some unresolved issues. Geogr. Compass 2007, 1, 695-712. [CrossRef]

10. Ashworth, G.; Kavaratzis, M. Beyond the logo: Brand management for cities. J. Brand Manag. 2009, 16, 520-531. [CrossRef]

11. Kavaratzis, M. From city marketing to city branding: Towards a theoretical framework for developing city brands. Place Brand. 2004, 1, 58-73. [CrossRef]

12. Ahn, Y.J.; Hyun, S.S.; Kim, I. City Residents' Perception of MICE city brand orientation and their brand citizenship behavior: A case study of busan, south Korea. Asia Pac. J. Tour. Res. 2016, 21, 328-353. [CrossRef]

13. Merrilees, B.; Miller, D.; Herington, C. City branding: A facilitating framework for stressed satellite cities. J. Bus. Res. 2013, 66, 37-44. [CrossRef] 
14. Han, M.; de Jong, M.; Cui, Z.; Xu, L.; Lu, H.; Sun, B. City branding in China's Northeastern region: How do cities reposition themselves when facing industrial decline and ecological modernization? Sustainability 2018, 10, 102. [CrossRef]

15. Benedek, I. Measuring place brand equity: A review of current models. J. Media Res. 2017, 10, 42-57. [CrossRef]

16. García, J.A.; Gómez, M.; Molina, A. A destination-branding model: An empirical analysis based on stakeholders. Tour. Manag. 2012, 33, 646-661. [CrossRef]

17. Dioko, L.D.A.N.; So, S.I.A. Branding destinations versus branding hotels in a gaming destination-Examining the nature and significance of co-branding effects in the case study of Macao. Int. J. Hosp. Manag. 2012, 31, 554-563. [CrossRef]

18. Pike, S.; Bianchi, C.; Kerr, G.; Patti, C. Consumer-based brand equity for Australia as a long-haul tourism destination in an emerging market. Int. Mark. Rev. 2010, 27, 434-449. [CrossRef]

19. Kavaratzis, M. Cities and their brands: Lessons from corporate branding. Place Brand. Public Dipl. 2009, 5, $26-37$. [CrossRef]

20. Kavaratzis, M.; Ashworth, G.J. City branding: An effective assertion of identity or a transitory marketing trick? Tijdschr. Voor Econ. Soc. Geogr. 2005, 96, 506-514. [CrossRef]

21. Parkerson, B.; Saunders, J. City branding: Can goods and services branding models be used to brand cities? Place Brand. 2005, 1, 242-264. [CrossRef]

22. Zhang, L.; Zhao, S.X. City branding and the olympic effect: A case study of Beijing. Cities 2009, 26, 245-254. [CrossRef]

23. Cardoso, L.; Dias, F.; de Araújo, A.F.; Andrés Marques, M.I. A destination imagery processing model: Structural differences between dream and favourite destinations. Ann. Tour. Res. 2019, 74, 81-94. [CrossRef]

24. Farquhar, P.H. Managing brand equity. J. Advert. Res. 1990, 30, 7-12.

25. Aaker, D.A. Managing Brand Equity: Capitalizing on the Value of a Brand Name; Free Press: New York, NY, USA; Maxwell Macmillan International: Toronto, ON, Canada; New York, NY, USA, 1991.

26. Keller, K.L. Conceptualizing, measuring, and managing customer-based brand equity. J. Mark. 1993, 57, 1. [CrossRef]

27. Yoo, B.; Donthu, N.; Lee, S. An examination of selected marketing mix elements and brand equity. J. Acad. Mark. Sci. 2000, 28, 195-211. [CrossRef]

28. Lassar, W.; Mittal, B.; Sharma, A. Measuring customer-based brand equity. J. Consum. Mark. 1995, 12, 11-19. [CrossRef]

29. Simon, C.J.; Sullivan, M.W. The measurement and determinants of brand equity: A financial approach. Mark. Sci. 1993, 12, 28-52. [CrossRef]

30. Morrison, M.; Eastburn, M. A study of brand equity in a commodity market. Australas. Mark. J. 2006, 14, $62-78$. [CrossRef]

31. Juga, J.; Juntunen, J.; Paananen, M. Impact of value-adding services on quality, loyalty and brand equity in the brewing industry. Int. J. Qual. Serv. Sci. 2018, 10, 61-71. [CrossRef]

32. Kimpakorn, N.; Tocquer, G. Service brand equity and employee brand commitment. J. Serv. Mark. 2010, 24, 378-388. [CrossRef]

33. Hazée, S.; Van Vaerenbergh, Y.; Armirotto, V. Co-creating service recovery after service failure: The role of brand equity. J. Bus. Res. 2017, 74, 101-109. [CrossRef]

34. Yoo, B.; Donthu, N. Developing and validating a multidimensional consumer-based brand equity scale. J. Bus. Res. 2001, 52,1-14. [CrossRef]

35. Pappu, R.; Cooksey, R.W.; Quester, P.G. Consumer-based brand equity: Improving the measurement-empirical evidence. J. Prod. Brand Manag. 2005, 14, 143-154. [CrossRef]

36. Šerić, M.; Gil-Saura, I. ICT, IMC, and brand equity in high-quality hotels of dalmatia: An analysis from guest perceptions. J. Hosp. Mark. Manag. 2012, 21, 821-851. [CrossRef]

37. Seric, M.; Gil-Saura, I. Perceptual and relational approach to hotel brand equity measurement, criticism, and challenges. In Routledge Handbook of Hospitality Marketing; Gursoy, D., Ed.; Routledge: London, UK, 2018; pp. 234-244. ISBN 978-1-315-44552-6.

38. Liu, M.T.; Wong, I.K.A.; Tseng, T.H.; Chang, A.W.Y.; Phau, I. Applying consumer-based brand equity in luxury hotel branding. J. Bus. Res. 2017, 81, 192-202. [CrossRef] 
39. Hanifah, R.D.; Wahyudi, A.S.; Nurbaeti, N. Influence of brand equity towards perceived value in 5 star hotel at Jakarta. In International Conference on Tourism, Gastronomy, and Tourist Destination (ICTGTD 2016); Abdullah, A.G., Rahmanita, M., Ingkadijaya, R., Suprina, R., Pramanik, P.D., Nahdlah, Z., Sugeng, N.W., Eds.; Atlantis Press: Paris, France, 2016; Volume 28, pp. 235-241.

40. Kayaman, R.; Arasli, H. Customer based brand equity: Evidence from the hotel industry. Manag. Serv. Qual. 2007, 17, 92-109. [CrossRef]

41. Ishaq, M.I.; Hussain, N.; Asim, A.I.; Cheema, L.J. Brand equity in the Pakistani hotel industry. Rae Rev. Adm. Empres. 2014, 54, 284-295. [CrossRef]

42. Surucu, O.; Ozturk, Y.; Okumus, F.; Bilgihan, A. Brand awareness, image, physical quality and employee behavior as building blocks of customer-based brand equity: Consequences in the hotel context. J. Hosp. Tour. Manag. 2019, 40, 114-124. [CrossRef]

43. Kim, H.-B.; Kim, W.G. The relationship between brand equity and firms' performance in luxury hotels and chain restaurants. Tour. Manag. 2005, 26, 549-560. [CrossRef]

44. Kam Fung so, K.; King, C. "When experience matters": Building and measuring hotel brand equity: The customers' perspective. Int. J. Contemp. Hosp. Manag. 2010, 22, 589-608. [CrossRef]

45. Han, S.H.; Nguyen, B.; Lee, T.J. Consumer-based chain restaurant brand equity, brand reputation, and brand trust. Int. J. Hosp. Manag. 2015, 50, 84-93. [CrossRef]

46. Bihamta, H.; Jayashree, S.; Rezaei, S.; Okumus, F.; Rahimi, R. Dual pillars of hotel restaurant food quality satisfaction and brand loyalty. Br. Food J. 2017, 119, 2597-2609. [CrossRef]

47. Majid, M.A.A.; Alias, M.A.M.; Samsudin, A.; Chik, C.T. Assessing customer-based brand equity ratings in family restaurant. Procedia Econ. Financ. 2016, 37, 183-189. [CrossRef]

48. Vatankhah, S.; Darvishi, M. An empirical investigation of antecedent and consequences of internal brand equity: Evidence from the airline industry. J. Air Transp. Manag. 2018, 69, 49-58. [CrossRef]

49. de Oliveira, D.; Caetano, M.S. Market strategy development and innovation to strengthen consumer-based equity: The case of Brazilian airlines. J. Air Transp. Manag. 2019, 75, 103-110. [CrossRef]

50. Chen, C.-F.; Tseng, W.-S. Exploring customer-based airline brand equity: Evidence from Taiwan. Transp. J. 2010, 49, 24-34.

51. Nel, J.; North, E.; Myburg, T.; Hern, L. A comparative study of customer-based brand equity across selected south African hotels. Int. Retail Mark. Rev. 2009, 5, 15-24.

52. Liu, C.-H.; Jiang, J.-F. Assessing the moderating roles of brand equity, intellectual capital and social capital in Chinese luxury hotels. J. Hosp. Tour. Manag. 2020, 43, 139-148. [CrossRef]

53. Šerić, M.; Gil-Saura, I.; Mollá-Descals, A. The Impact of Integrated Marketing Communications on Hotel Brand Equity: Does National Culture Matter? In Handbook of Research on Global Hospitality and Tourism Research; Camilo, A., Ed.; IGI Global: Boston, MA, USA, 2015; ISBN 9781466686076.

54. Xu, J.B.; Chan, A. A conceptual framework of hotel experience and customer-based brand equity Some research questions and implications. Int. J. Contemp. Hosp. Manag. 2010, 22, 174-193.

55. Martinez, R.; Nishiyama, N. Enhancing customer-based brand equity through CSR in the hospitality sector. Int. J. Hosp. Tour. Adm. 2019, 20, 329-353. [CrossRef]

56. Seric, M.; Gil-Saura, I. Understanding brand equity in hotel firms. What is the role of brand loyalty and satisfaction? Int. J. Contemp. Hosp. Manag. 2019, 31, 3526-3546. [CrossRef]

57. Šerić, M.; Gil-Saura, I.; Ruiz-Molina, M.E. How can integrated marketing communications and advanced technology influence the creation of customer-based brand equity? Evidence from the hospitality industry. Int. J. Hosp. Manag. 2014, 39, 144-156. [CrossRef]

58. Kim, W.G.; Kim, H.B. Measuring customer-based restaurant brand equity: Investigating the relationship between brand equity and firms' performance. Cornell Hotel Restaur. Adm. Q. 2004, 45, 115-131. [CrossRef]

59. Namkung, Y.; Jang, S. Effects of restaurant green practices on brand equity formation: Do green practices really matter? Int. J. Hosp. Manag. 2013, 33, 85-95. [CrossRef]

60. Tranfield, D.; Denyer, D.; Smart, P. Towards a methodology for developing evidence-informed management knowledge by means of systematic review. Br. J. Manag. 2003, 14, 207-222. [CrossRef]

61. Oguztimur, S.; Akturan, U. Synthesis of city branding literature (1988-2014) as a research domain. Int. J. Tour. Res. 2016, 18, 357-372. [CrossRef]

62. Kasapi, I.; Cela, A. Destination branding: A review of the city branding literature. Mediterr. J. Soc. Sci. 2017, 8, 129-142. [CrossRef] 
63. Lucarelli, A.; Olof Berg, P. City branding: A state-of-the-art review of the research domain. J. Place Manag. Dev. 2011, 4, 9-27. [CrossRef]

64. Niñerola, A.; Sánchez-Rebull, M.V.; Hernández-Lara, A.B. Tourism research on sustainability: A bibliometric analysis. Sustainability 2019, 11, 1377. [CrossRef]

65. Luo, J.; Ji, C.; Qiu, C.; Jia, F. Agri-food supply chain management: Bibliometric and content analyses. Sustainability 2018, 10, 1573. [CrossRef]

66. Hedin, B.; Katzeff, C.; Eriksson, E.; Pargman, D. A systematic review of digital behaviour change interventions for more sustainable food consumption. Sustainability 2019, 11, 2638. [CrossRef]

67. Głąsska, D.; Guzek, D.; Groele, B.; Gutkowska, K. Fruit and vegetable intake and mental health in adults: A systematic review. Nutrients 2020, 12, 115. [CrossRef] [PubMed]

68. Liberati, A.; Altman, D.G.; Tetzlaff, J.; Mulrow, C.; Gøtzsche, P.C.; Ioannidis, J.P.A.; Clarke, M.; Devereaux, P.J.; Kleijnen, J.; Moher, D. The PRISMA statement for reporting systematic reviews and meta-analyses of studies that evaluate health care interventions: Explanation and elaboration. PLoS Med. 2009, 6, e1-e34. [CrossRef] [PubMed]

69. Lemus-Aguilar, I.; Morales-Alonso, G.; Ramirez-Portilla, A.; Hidalgo, A. Sustainable business models through the lens of organizational design: A systematic literature review. Sustainability 2019, 11, 5379. [CrossRef]

70. Gregorio, V.F.; Pié, L.; Terceño, A. A systematic literature review of bio, green and circular economy trends in publications in the field of economics and business management. Sustainability 2018, 10, 4232. [CrossRef]

71. Corallo, A.; Lazoi, M.; Striani, F. Process mining and industrial applications: A systematic literature review. Knowl. Process Manag. 2020, 27, 225-233. [CrossRef]

72. How to Evaluate the Impact of Lean Supply Chain Strategy: A Systematic Literature Review|Request PDF. Available online: https://www.researchgate.net/publication/332154035_How_to_evaluate_the_impact_of_ lean_supply_chain_strategy_a_systematic_literature_review (accessed on 23 August 2020).

73. Harsanto, B.; Permana, C. Understanding sustainability-oriented innovation (SOI) using network perspective in asia pacific and ASEAN: A systematic review. J. ASEAN Stud. 2019, 7, 1-17. [CrossRef]

74. Wohlin, C. Guidelines for snowballing in systematic literature studies and a replication in software engineering. In Proceedings of the 18th International Conference on Evaluation and Assessment in Software Engineering, London, UK, 13-14 May 2014; pp. 1-10. [CrossRef]

75. Huerta-Álvarez, R.; Cambra-Fierro, J.J.; Fuentes-Blasco, M. The interplay between social media communication, brand equity and brand engagement in tourist destinations: An analysis in an emerging economy. J. Destin. Mark. Manag. 2020, 16, 100413. [CrossRef]

76. Dedeoğlu, B.B.; Niekerk, M.; Van Weinland, J.; Celuch, K. Re-conceptualizing customer-based destination brand equity. J. Destin. Mark. Manag. 2019, 11, 211-230. [CrossRef]

77. Tran, V.T.; Nguyen, N.P.; Tran, P.T.K.; Tran, T.N.; Huynh, T.T.P. Brand equity in a tourism destination: A case study of domestic tourists in Hoi an city, Vietnam. Tour. Rev. 2019, 74, 704-720. [CrossRef]

78. Tarman, H.A.; Ari, A. Leveraging brand equity by applying brand communication and forming city branding based on unique selling proposition (a case of crafts city). Int. J. Bus. Adm. Stud. 2019, 5, 75-84.

79. Rani, P. Customer-based brand equity index of Kurukshetra. J. Tour. Anal. 2019, 26, 48-61. [CrossRef]

80. Subagyo, H. The effect of destination brand awareness on destination brand loyalty through brand image, perceived brand quality, brand value and brand satisfaction in banyuwangi city as a tourism objective. Arch. Bus. Res. 2019, 7, 211-225.

81. Gómez, M.; Fernández, A.C.; Molina, A.; Aranda, E. City branding in European capitals: An analysis from the visitor perspective. J. Destin. Mark. Manag. 2018, 7, 190-201. [CrossRef]

82. Kotsi, F.; Pike, S.; Gottlieb, U. Consumer-based brand equity (CBBE) in the context of an international stopover destination: Perceptions of dubai in France and Australia. Tour. Manag. 2018, 69, 297-306. [CrossRef]

83. Duman, T.; Ozbal, O.; Duerod, M. The role of affective factors on brand resonance: Measuring customer-based brand equity for the Sarajevo brand. J. Destin. Mark. Manag. 2018, 8, 359-372. [CrossRef]

84. Kazemi, M.; Hesam, M.; Rad, M.S.; Cheraghi, M. The evaluation of tourism destination brand equity from the female tourist's perspectives (study of 16 cities in Iran). Geoj. Tour. Geosites 2018, 21, 112-122.

85. Tasci, A.D.A. Testing the cross-brand and cross-market validity of a consumer-based brand equity (CBBE) model for destination brands. Tour. Manag. 2018, 65, 143-159. [CrossRef]

86. Stojanovic, I.; Andreu, L.; Curras-Perez, R. Effects of the intensity of use of social media on brand equity: An empirical study in a tourist destination. Eur. J. Manag. Bus. Econ. 2018, 27, 83-100. [CrossRef] 
87. Kim, H.-K.; Lee, T.J. Brand equity of a tourist destination. Sustainability 2018, 10, 431. [CrossRef]

88. Lee, K. City brand competitiveness: Exploring structural relationships among city brand equity elements in China. Int. J. Bus. Econ. Res. 2017, 6, 32. [CrossRef]

89. Tasseven, O.; Ardahanlioglu, E. Cultural destination brand equity: Case study of istanbul. Int. J. Econ. Perspect. 2017, 11, 136-159.

90. Vinh, T.T.; Nga, V.T.Q.; Nguyen, N.P. The causal relationships between components of customer-based brand equity for a destination: Evidence from south Korean tourists in Danang City, Vietnam. Asian Econ. Financ. Rev. 2017, 7, 358-367. [CrossRef]

91. Pirnar, I.; Igneci, M.; Tutuncuoglu, M. Trends and applications in city branding: A case study in Izmir. Mark. Brand. Res. 2017, 4, 25-32. [CrossRef]

92. Esmaili, S.; Rezaei, N.; Abbasi, R.; Eskandari, S. The impact of marketing mix on perceived value, destination image and loyalty of tourists (Case Study: Khalkhal City, Iran). Mod. Appl. Sci. 2017, 11, 96. [CrossRef]

93. Alizadeh, K.; Khamseh, P.; Ettehadi, S. Explanation of the city brand equity role on specialists' identity and immigration (Case of study: Mashhad). A Q. J. Urban Econ. Manag. 2016, 4, 49-61.

94. Salehzadeh, R.; Khazaei Pool, J.; Soleimani, S. Brand personality, brand equity and revisit intention: An empirical study of a tourist destination in Iran. Tour. Rev. 2016, 71, 205-218. [CrossRef]

95. Peighambari, K.; Sattari, S.; Foster, T.; Wallström, A. Two tales of one city: Image versus identity. Place Brand. Public Dipl. 2016, 12, 268-284. [CrossRef]

96. Kashif, M.; Samsi, S.Z.M.; Sarifuddin, S. Brand equity of lahore fort as a tourism destination brand. Rev. Adm. Empres. 2015, 55, 432-444. [CrossRef]

97. Wong, P.P.W.; Teoh, K. The influence of destination competitiveness on customer-based brand equity. J. Destin. Mark. Manag. 2015, 4, 206-212. [CrossRef]

98. Jørgensen, O.H. Developing a city brand balance sheet-using the case of Horsens, Denmark. Place Brand. Public Dipl. 2015, 11, 148-160. [CrossRef]

99. Kladou, S.; Kehagias, J. Assessing destination brand equity: An integrated approach. J. Destin. Mark. Manag. 2014, 3, 2-10. [CrossRef]

100. Andéhn, M.; Kazeminia, A.; Lucarelli, A.; Sevin, E. User-generated place brand equity on Twitter: The dynamics of brand associations in social media. Place Brand. Public Dipl. 2014, 10, 132-144. [CrossRef]

101. Zenker, S. Measuring place brand equity with the advanced brand concept map (aBCM) method. Place Brand. Public Dipl. 2014, 10, 158-166. [CrossRef]

102. Abbasi, M.; Roosta, A. Assessment of factors affecting destination brand value in tourism industry (Shiraz city). Int. J. Sci. Manag. Dev. 2014, 2, 518-528.

103. Chan, C. Applying linear programming in city brand equity concept: A case study of Hong Kong. Int. J. Tour. Sci. 2014, 14, 1-22. [CrossRef]

104. Shafranskaya, I.; Potapov, D. An empirical study of consumer-based city brand equity from signalling theory perspective. Place Brand. Public Dipl. 2014, 10, 117-131. [CrossRef]

105. Hakala, U.; Lemmetyinen, A. "Culture is the message": The status of cultural capital and its effect on a city's brand equity. Place Brand. Public Dipl. 2013, 9, 5-16. [CrossRef]

106. Yuwo, H.; Ford, J.; Purwanegara, M.S. Customer-based brand equity for a tourism destination (CBBETD): The specieic case of bandung city. Organ. Mark. Emerg. Econ. 2013, 4. [CrossRef]

107. Lemmetyinen, A.; Go, F.; Luonila, M. The relevance of cultural production-pori jazz-in boosting place brand equity. Place Brand. Public Dipl. 2013, 9, 164-181. [CrossRef]

108. Al-Azzam, A.F. Analysis of the antecedents of customer-based brand equity and its application to multiple destinations. Interdiscip. J. Contemp. Res. Bus. 2013, 5, 71-90.

109. Florek, M. City brand equity as one of the city sustainable development indicator. Transform. Bus. Econ. 2012, 11, 385-405.

110. Boo, S.; Busser, J.; Baloglu, S. A model of customer-based brand equity and its application to multiple destinations. Tour. Manag. 2009, 30, 219-231. [CrossRef]

111. Bianchi, C.; Pike, S. Antecedents of destination brand loyalty for a long-haul market: Australia's destination loyalty among chilean travelers. J. Travel Tour. Mark. 2011, 28, 736-750. [CrossRef]

112. Almeyda-Ibáñez, M.; George, B. Place branding in tourism: A review of theoretical approaches and management practices. Tour. Manag. Stud. 2017, 13, 10-19. [CrossRef] 
113. Zanfardini, M.; Tamagni, L.; Gutauskas, A. Costumer-based brand equity for tourism destinations in Patagonia. Catalan J. Commun. Cult. Stud. 2011, 3, 253-271. [CrossRef]

114. Saeed, M.; Shafique, I. Customer-based brand equity and destination visit behaviour in the tourism industry: The contingent role of social media. Qual. Quant. 2019, 1-22. [CrossRef]

115. Bianchi, C.; Pike, S.; Lings, I. Investigating attitudes towards three south American destinations in an emerging long haul market using a model of consumer-based brand equity (CBBE). Tour. Manag. 2014, 42, 215-223. [CrossRef]

116. Konecnik, M. Croatian-based brand equity for slovenia as a tourism destination. Econ. Bus. Rev. Cent. South East. Eur. 2006, 8, 83-108.

117. Almeyda, M.; George, B. Customer-based brand equity for tourist destinations: A comparison of equities of puerto rico and the USA virgin islands. J. Spat. Organ. Dyn. 2019, 8, 148-172.

118. Gartner, W.C.; Ruzzier, M.K. Tourism destination brand equity dimensions. J. Travel Res. 2011, 50, $471-481$. [CrossRef]

119. Gómez, M.; Lopez, C.; Molina, A. A model of tourism destination brand equity: The case of wine tourism destinations in Spain. Tour. Manag. 2015, 51, 210-222. [CrossRef]

120. Kim, S.H.; Han, H.S.; Holland, S.; Byon, K.K. Structural relationships among involvement, destination brand equity, satisfaction and destination visit intentions: The case of Japanese outbound travelers. J. Vacat. Mark. 2009, 15, 349-365. [CrossRef]

121. Zavattaro, S.M.; Daspit, J.J.; Adams, F.G. Assessing managerial methods for evaluating place brand equity: A qualitative investigation. Tour. Manag. 2015, 47, 11-21. [CrossRef]

122. Chan, C.S.; Peters, M.; Marafa, L.M. An assessment of place brand potential: Familiarity, favourability and uniqueness. J. Place Manag. Dev. 2016, 9, 269-288. [CrossRef]

123. Konecnik, M.; Gartner, W.C. Customer-based brand equity for a destination. Ann. Tour. Res. 2007, 34, 400-421. [CrossRef]

124. Kim, S.; Schuckert, M.; Im, H.H.; Elliot, S. An interregional extension of destination brand equity: From Hong Kong to Europe. J. Vacat. Mark. 2017, 23, 277-294. [CrossRef]

125. Yousaf, A.; Amin, I. Can customer based brand equity help destinations to stay in race? An empirical study of kashmir valley. Tour. Hosp. Manag. 2017, 23, 251-266. [CrossRef]

126. Jacobsen, B.P. Investor-based place brand equity: A theoretical framework. J. Place Manag. Dev. 2009, 2, 70-84. [CrossRef]

127. Simon, A. The Anholt-GMI city brands index how the world sees the world's cities. Place Brand. 2006, 2, $18-31$.

128. Lucarelli, A. Unraveling the complexity of "city brand equity": A three-dimensional framework. J. Place Manag. Dev. 2012, 5, 231-252. [CrossRef]

129. Yousaf, A.; Amin, I.; Gupta, A. Conceptualising tourist based brand-equity pyramid: An application of keller brand pyramid model to destinations. Tour. Hosp. Manag. 2017, 23, 119-137. [CrossRef]

130. Papp-Vary, A. The Anholt-GMI city brand hexagon and the saffron european city brand barometer: A comparative study. Reg. Bus. Stud. 2011, 3, 555-562.

131. Chan, C.S.; Marafa, L.M. The green branding of Hong Kong: Visitors' and residents' perceptions. J. Place Manag. Dev. 2016, 9, 289-312. [CrossRef]

132. Chan, C.S.; Marafa, L.M.; Van Den Bosch, C.C.K.; Randrup, T.B. Starting conditions for the green branding of a city. J. Destin. Mark. Manag. 2018, 10,10-24. [CrossRef]

133. Ferns, B.H.; Walls, A. Enduring travel involvement, destination brand equity, and travelers' visit intentions: A structural model analysis. J. Destin. Mark. Manag. 2012, 1, 27-35. [CrossRef]

134. Tasci, A.D.A.; Gartner, W.C.; Cavusgil, S.T. Measurement of destination brand bias using a quasi-experimental design. Tour. Manag. 2007, 28, 1529-1540. [CrossRef]

135. Cai, L.A. Cooperative branding for rural destinations. Ann. Tour. Res. 2002, 29, 720-742. [CrossRef]

136. Beerli, A.; Martín, J.D. Tourists' characteristics and the perceived image of tourist destinations: A quantitative analysis-a case study of Lanzarote, Spain. Tour. Manag. 2004, 25, 623-636. [CrossRef]

137. Barreda, A. Creating brand equity when using travel-related online social network web sites. J. Vacat. Mark. 2014, 20, 365-379. [CrossRef]

138. Upshaw, L. Building Brand Identity: A Strategy for Success in a Hostile Marketplace; John Wiley \& Sons: New York, NY, USA, 1995; ISBN 978-0-471-04220-4. 
139. Aaker, D.A. Measuring brand equity across products and markets. Calif. Manag. Rev. 1996, 38, $102-120$. [CrossRef]

140. Lee, T.H. A structural model to examine how destination image, attitude, and motivation affect the future behavior of tourists. Leis. Sci. 2009, 31, 215-236. [CrossRef]

141. Camprubí, R.; Guia, J.; Comas, J. The new role of tourists in destination image formation. Curr. Issues Tour. 2013, 16, 203-209. [CrossRef]

142. Baloglu, S. A path analytic model of visitation intention involving information sources, socio-psychological motivations, and destination image. J. Travel Tour. Mark. 2000, 8, 81-90. [CrossRef]

143. Wang, C.Y.; Hsu, M.K. The relationships of destination image, satisfaction, and behavioral intentions: An integrated model. J. Travel Tour. Mark. 2010, 27, 829-843. [CrossRef]

144. Dann, G.M.S. Tourists' images of a destination an alternative analysis. J. Travel Tour. Mark. 1996, 5, 41-55. [CrossRef]

145. Huete Alcocer, N.; López Ruiz, V.R. The role of destination image in tourist satisfaction: The case of a heritage site. Econ. Res. Istraz. 2019, 33, 2444-2461. [CrossRef]

146. Beerli, A.; Martín, J.D. Factors influencing destination image. Ann. Tour. Res. 2004, 31, 657-681. [CrossRef]

147. Zeithaml, V.A. Consumer perceptions of price, quality, and value: A means-end model and synthesis of evidence. J. Mark. 1988, 52, 2-22. [CrossRef]

148. Keller, K.L. Brand synthesis: The multidimensionality of brand knowledge. J. Consum. Res. 2003, 29, 595-600. [CrossRef]

149. Fensel, A.; Toma, I.; García, J.M.; Stavrakantonakis, I.; Fensel, D. Enabling customers engagement and collaboration for small and medium-sized enterprises in ubiquitous multi-channel ecosystems. Comput. Ind. 2014, 65, 891-904. [CrossRef]

150. Chiesura, A. The role of urban parks for the sustainable city. Landsc. Urban Plan. 2004, 68, 129-138. [CrossRef]

151. Fok, K.W.K.; Law, W.W.Y. City re-imagined: Multi-stakeholder study on branding Hong Kong as a city of greenery. J. Environ. Manag. 2018, 206, 1039-1051. [CrossRef] [PubMed]

152. Gulsrud, N.M.; Gooding, S.; van den Bosch, C.C.K. Green space branding in Denmark in an era of neoliberal governance. Urban Urban Green. 2013, 12, 330-337. [CrossRef]

153. Chan, C.S.; Marafa, L.M. How a green city brand determines the willingness to stay in a city: The case of Hong Kong. J. Travel Tour. Mark. 2017, 34, 719-731. [CrossRef]

154. Gartner, W.C. Image formation process. J. Travel Tour. Mark. 1994, 2, 191-216. [CrossRef]

155. Song, Z.; Su, X.; Li, L. The indirect effects of destination image on destination loyalty intention through tourist satisfaction and perceived value: The bootstrap approach. J. Travel Tour. Mark. 2013, 30, 386-409. [CrossRef]

156. Trembath, R.; Romaniuk, J.; Lockshin, L. Building the destination brand: An empirical comparison of two approaches. J. Travel Tour. Mark. 2011, 28, 804-816. [CrossRef]

157. Jago, L.; Chalip, L.; Brown, G.; Mules, T.; Ali, S. Building events into destination branding: Insights from experts. Event Manag. 2008, 8, 3-14. [CrossRef]

158. Rosenbaum-Elliott, R.; Percy, L.; Pervan, S. Strategic Brand Management; Oxford University Press: Oxford, UK, 2015.

159. Chaudhuri, A.; Holbrook, M.B. The chain of effects from brand trust and brand affect to brand performance: The role of brand loyalty. J. Mark. 2001, 65, 81-93. [CrossRef]

160. Kolotouchkina, O.; Seisdedos, G. The urban cultural appeal matrix: Identifying key elements of the cultural city brand profile using the example of madrid. Place Brand. Public Dipl. 2016, 12, 59-67. [CrossRef]

161. Karabağ, S.F.; Yavuz, M.C.; Berggren, C. The impact of festivals on city promotion: A comparative study of turkish and swedish festivals. Tourism 2011, 59, 447-464.

162. Sari, C.T.; Subagyo, H. SWOT analysis of art performance as city promotional strategy in Yogyakarta. J. Educ. Cult. Soc. 2020, 11, 370-378. [CrossRef]

163. Felsenstein, D.; Fleischer, A. local festivals and tourism promotion: The role of public assistance and visitor expenditure. J. Travel Res. 2003, 41, 385-392. [CrossRef]

164. Mazdon, L. Transnational 'French' cinema: The cannes film festival. Mod. Contemp. Fr. 2007, 15, 9-20. [CrossRef]

165. Kusumawati, A. Integrating the concepts of city branding and tourism event on behavioural intention in domestic urban tourism. Russ. J. Agric. Socio Econ. Sci. 2017, 65, 155-161. [CrossRef] 
166. UNESCO. World Heritage Centre-World Heritage List. Available online: https://whc.unesco.org/en/list/ (accessed on 25 August 2020).

167. UNESCO. World Heritage Centre-World Heritage. Available online: https://whc.unesco.org/en/about/ (accessed on 25 August 2020).

168. Organization of World Heritage Cities. Study of Heritage and Sustainable Tourism; Organization of World Heritage Cities: Quebec City, QC, Canada, 2019; pp. 1-46. Available online: https://owhc2019krakow.org/ wp-content/uploads/2019/05/sustainable-tourism.pdf (accessed on 25 August 2020).

169. Santa-Cruz, F.G.; López-Guzmán, T. Culture, tourism and world heritage sites. Tour. Manag. Perspect. 2017, 24, 111-116. [CrossRef]

170. Liu, Y. De place in practice: Major event and city branding: An evaluation of liverpool as the 2008 European capital of culture. J. Place Manag. Dev. 2015, 8, 147-162. [CrossRef]

(C) 2020 by the author. Licensee MDPI, Basel, Switzerland. This article is an open access article distributed under the terms and conditions of the Creative Commons Attribution (CC BY) license (http://creativecommons.org/licenses/by/4.0/). 